\title{
Cutting and pasting in the Torelli group
}

\author{
ANDREW PUTMAN
}

\begin{abstract}
We introduce machinery to allow "cut-and-paste"-style inductive arguments in the Torelli subgroup of the mapping class group. In the past these arguments have been problematic because restricting the Torelli group to subsurfaces gives different groups depending on how the subsurfaces are embedded. We define a category TSur whose objects are surfaces together with a decoration restricting how they can be embedded into larger surfaces and whose morphisms are embeddings which respect the decoration. There is a natural "Torelli functor" on this category which extends the usual definition of the Torelli group on a closed surface. Additionally, we prove an analogue of the Birman exact sequence for the Torelli groups of surfaces with boundary and use the action of the Torelli group on the complex of curves to find generators for the Torelli group. For genus $g \geq 1$ only twists about (certain) separating curves and bounding pairs are needed, while for genus $g=0$ a new type of generator (a "commutator of a simply intersecting pair") is needed. As a special case, our methods provide a new, more conceptual proof of the classical result of Birman and Powell which says that the Torelli group on a closed surface is generated by twists about separating curves and bounding pairs.
\end{abstract}

57S05; 20F05, 57M07, 57N05

\section{Introduction}

Let $\Sigma_{g, n}$ be a genus $g$ surface with $n$ boundary components (we will often omit the $n$ if it equals 0 and omit both subscripts if they are unimportant). The mapping class group $\operatorname{Mod}\left(\Sigma_{g, n}\right)$ is the group of orientation-preserving homeomorphisms of $\Sigma_{g, n}$ which fix the boundary pointwise modulo isotopies fixing the boundary pointwise. The action of $\operatorname{Mod}\left(\Sigma_{g, n}\right)$ on $\mathrm{H}_{1}\left(\Sigma_{g, n} ; \mathbb{Z}\right)$ preserves the algebraic intersection form. If $n \leq 1$, then this is a nondegenerate alternating form, so in this case the action yields a representation $\operatorname{Mod}\left(\Sigma_{g, n}\right) \rightarrow \operatorname{Sp}(2 g, \mathbb{Z})$, which is well-known to be surjective. Its kernel is the Torelli group $\mathcal{I}\left(\Sigma_{g, n}\right)$. Summarizing, for $n \leq 1$ we have an exact sequence

$$
1 \longrightarrow \mathcal{I}\left(\Sigma_{g, n}\right) \longrightarrow \operatorname{Mod}\left(\Sigma_{g, n}\right) \longrightarrow \operatorname{Sp}(2 g, \mathbb{Z}) \longrightarrow 1
$$


The group $\mathcal{I}\left(\Sigma_{g, n}\right)$ plays an important role in both algebraic geometry and low dimensional topology. For a survey of the Torelli group (especially the remarkable results of Johnson which appear in $[14 ; 15 ; 16])$, see [13].

If $i: \Sigma \hookrightarrow \Sigma^{\prime}$ is an embedding, then there is an induced map $i_{*}: \operatorname{Mod}(\Sigma) \rightarrow \operatorname{Mod}\left(\Sigma^{\prime}\right)$. Namely, if $f \in \operatorname{Mod}(\Sigma)$, then $i_{*}(f)$ equals $f$ on $\Sigma \subset \Sigma^{\prime}$ and the identity elsewhere. In fancier language, if Sur is the category whose objects are surfaces and whose morphisms are embeddings, then Mod is a functor from Sur to the category of groups and homomorphisms. These morphisms are fundamental tools in the study of $\operatorname{Mod}(\Sigma)$ (they allow proofs by "cutting and inducting"). In this paper, we develop such technology for the Torelli group.

This technology has been problematic in the past because the naive definition of the Torelli group on a surface with boundary simply does not work. Indeed, no single definition of $\mathcal{I}\left(\Sigma_{g, n}\right)$ for $n>1$ satisfies the following two properties, which are the minimum needed for inductive proofs:

- $\mathcal{I}$ should be functorial in the sense that if $i: \Sigma \hookrightarrow \Sigma^{\prime}$ is an embedding, then $i_{*}(\mathcal{I}(\Sigma)) \subset \mathcal{I}\left(\Sigma^{\prime}\right)$.

- $\mathcal{I}$ should be closed under restriction in the sense that if $i: \Sigma \hookrightarrow \Sigma^{\prime}$ is an embedding, then $\mathcal{I}(\Sigma)=i_{*}^{-1}\left(\mathcal{I}\left(\Sigma^{\prime}\right)\right)$.

To see that these properties are mutually contradictory and to explain our solution, we need some more concepts. For a simple closed curve $\gamma$, let $T_{\gamma}$ be the right Dehn twist about $\gamma$. A curve $\gamma$ is a separating curve if it separates the surface into two pieces (for instance, the curve $\gamma_{1}$ in Figure 1.c). A pair of disjoint non-isotopic simple closed curves $\left\{\gamma, \gamma^{\prime}\right\}$ form a bounding pair if neither $\gamma$ nor $\gamma^{\prime}$ separate the surface but $\gamma \cup \gamma^{\prime}$ does (for instance, the pair $\left\{\gamma_{2}, \gamma_{3}\right\}$ in Figure 1.c). It is not hard to see that $T_{\gamma} \in \mathcal{I}\left(\Sigma_{g}\right)$ if and only if $\gamma$ is a separating curve, and similarly if $\left\{\gamma, \gamma^{\prime}\right\}$ is a bounding pair in $\Sigma_{g}$, then $T_{\gamma} T_{\gamma^{\prime}}^{-1} \in \mathcal{I}\left(\Sigma_{g}\right)$ (such a mapping class will be called a twist about a bounding pair).

Now assume that we have a definition of the Torelli group which is both functorial and closed under restriction, and consider Figure 1, which shows two embeddings of $\Sigma_{0,4}$ into closed surfaces. The twist $T_{\gamma}$ is a twist about a separating curve in $\mathcal{I}\left(\Sigma_{4}\right)$, so since $\mathcal{I}$ is closed under restriction, we must have $T_{\gamma} \in \mathcal{I}\left(\Sigma_{0,4}\right)$. However, by functoriality we would then have $T_{\gamma} \in \mathcal{I}\left(\Sigma_{2}\right)$. Since $\gamma$ is not a separating curve in $\Sigma_{2}$, this is a contradiction.

One difference between the embeddings $\Sigma_{0,4} \hookrightarrow \Sigma_{2}$ and $\Sigma_{0,4} \hookrightarrow \Sigma_{4}$ depicted in Figure 1 is that the partitions

$$
\left\{\partial S \mid S \text { is a component of } \Sigma_{i} \backslash \operatorname{Int}\left(\Sigma_{0,4}\right)\right\}
$$




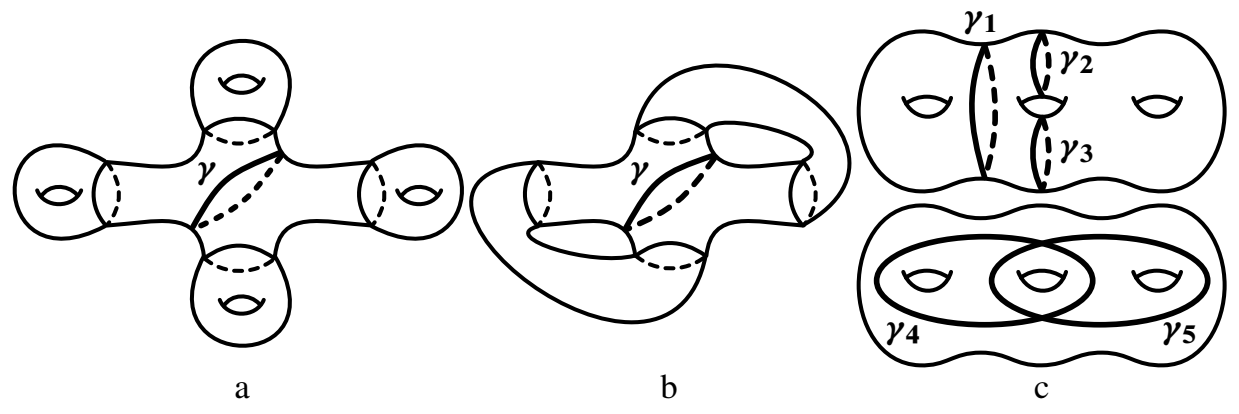

Figure 1: a, b: Two different embeddings of $\Sigma_{0,4}$ into larger surfaces c: A separating curve, a bounding pair and a simply intersecting pair

of the boundary components of $\Sigma_{0,4}$ are different. It turns out that this additional piece of data is exactly what we need to distinguish between the behavior of the Torelli groups under different embeddings. We will call a pair $(\Sigma, P)$ consisting of a surface $\Sigma$ and a partition $P$ of the boundary components of $\Sigma$ a partitioned surface (we think of each partition element as a "chunk" of the boundary to which we can attach pieces). We will construct a category TSur whose elements are partitioned surfaces and whose morphisms are embeddings which "respect the partitions" (see Section 3.2 for the precise definition of the morphisms). We will also define on a partitioned surface $(\Sigma, P)$ a "homology group" $\mathrm{H}_{1}^{P}(\Sigma)$ (see Section 3.1). Our main theorem will then be the following:

Theorem Summary 1.1 There is a functor $\mathcal{I}$ from the category TSur to the category of groups and homomorphisms (see Corollary 3.7) which satisfies the following properties:

- If $i: \Sigma \hookrightarrow \Sigma_{g}$ is an embedding and

$$
P=\left\{\partial S \mid S \text { is a component of } \Sigma_{g} \backslash \operatorname{Int}(\Sigma)\right\},
$$

then $\mathcal{I}(\Sigma, P)=i_{*}^{-1}\left(\mathcal{I}\left(\Sigma_{g}\right)\right.$ ) (see Theorem 3.3).

- For a partitioned surface $(\Sigma, P)$, the group $\mathcal{I}(\Sigma, P)$ equals the subgroup of $\operatorname{Mod}(\Sigma)$ acting trivially on $\mathrm{H}_{1}^{P}(\Sigma)$ (see Theorem 3.3).

- $\mathcal{I}$ is closed under restriction in the following sense : if $\left(\Sigma^{\prime}, P^{\prime}\right)$ is a partitioned surface and $i: \Sigma \hookrightarrow \Sigma^{\prime}$ is an embedding, then there is some partition $P$ of the boundary components of $\Sigma$ so that $\mathcal{I}(\Sigma, P)=i_{*}^{-1}\left(\mathcal{I}\left(\Sigma^{\prime}, P^{\prime}\right)\right.$ ) (see Theorem 3.8).

Now, the mapping class groups on surfaces with differing numbers of boundary components are related by the fundamental Birman exact sequences (see [2; 4] and Section 
2.1). One version of this, due to Johnson [14], is that for $n \geq 1$ and $(g, n) \neq(1,1)$, we have an exact sequence

$$
1 \longrightarrow \pi_{1}\left(U \Sigma_{g, n-1}\right) \longrightarrow \operatorname{Mod}\left(\Sigma_{g, n}\right) \longrightarrow \operatorname{Mod}\left(\Sigma_{g, n-1}\right) \longrightarrow 1,
$$

where $U \Sigma_{g, n-1}$ is the unit tangent bundle of $\Sigma_{g, n-1}$, the map

$$
\operatorname{Mod}\left(\Sigma_{g, n}\right) \longrightarrow \operatorname{Mod}\left(\Sigma_{g, n-1}\right)
$$

is induced by "gluing a disc to a boundary component", and the map

$$
\pi_{1}\left(U \Sigma_{g, n-1}\right) \rightarrow \operatorname{Mod}\left(\Sigma_{g, n}\right)
$$

is induced by "pushing the boundary component around curves". We construct a similar exact sequence for the Torelli group:

Theorem 1.2 Let $\left(\Sigma_{g, n}, P\right)$ be a partitioned surface with $n \geq 1$ and $(g, n) \neq(1,1)$, and let $b$ be a boundary component of $\Sigma_{g, n}$ contained in a set $p \in P$. Consider the map $i: \Sigma_{g, n} \hookrightarrow \Sigma_{g, n-1}$ induced by gluing a disc to $b$, and let $P^{\prime}$ be the partition of the boundary components of $\Sigma_{g . n-1}$ induced by $i$. There is then an exact sequence

$$
1 \longrightarrow K \longrightarrow \mathcal{I}\left(\Sigma_{g, n}, P\right) \longrightarrow \mathcal{I}\left(\Sigma_{g, n-1}, P^{\prime}\right) \longrightarrow 1
$$

with $K$ equal to the following:

- If $p=\{b\}$, then $K=\pi_{1}\left(U \Sigma_{g, n-1}\right)$.

- If $p \neq\{b\}$, then $K$ is isomorphic to the kernel of the natural map $\pi_{1}\left(\Sigma_{g, n-1}\right) \rightarrow$ $\mathrm{H}_{1}^{P^{\prime}}\left(\Sigma_{g, n-1}\right)$ (recall that $\mathrm{H}_{1}^{P^{\prime}}\left(\Sigma_{g, n-1}\right)$ is the "homology group" discussed in Theorem Summary 1.1).

See Section 4 for a discussion of the manner in which the kernel $K$ embeds into $\pi_{1}\left(U \Sigma_{g, n-1}\right)$.

Finally, we combine the machinery we have developed with action of the Torelli group on the complex of curves $\mathcal{C}(\Sigma)$ (see Section 5) to determine generators for $\mathcal{I}(\Sigma, P)$. We define " $P$-separating curves" and " $P$-bounding pairs" to be separating curves and bounding pairs which "respect the partition $P$ " (see the end of Section 3.1 for a precise definition). Our first theorem is then the following:

Theorem 1.3 For any partitioned surface $\left(\Sigma_{g, n}, P\right)$ with $g \geq 1$, the group $\mathcal{I}\left(\Sigma_{g, n}, P\right)$ is generated by twists about $P$-separating curves and twists about $P$-bounding pairs.

As a special case of this theorem, we obtain a new proof of the following classical theorem of Birman and Powell [3; 19]: 
Corollary 1.4 For a surface $\Sigma_{g, n}$ with $n \leq 1$, the group $\mathcal{I}\left(\Sigma_{g, n}\right)$ is generated by twists about separating curves and bounding pairs.

Our proof of Theorem 1.3 makes use of a basic result of Armstrong [1] (see Section 2.2) which says that if a group $G$ acts nicely on a simply-connected simplicial complex $X$, then $G$ is generated by elements which stabilize vertices of $X$ if and only if $X / G$ is simply connected. We prove that $\mathcal{C}\left(\Sigma_{g}\right) / \mathcal{I}\left(\Sigma_{g}\right)$ is simply connected for $g \geq 2$, so Armstrong's theorem says that $\mathcal{I}\left(\Sigma_{g}\right)$ is generated by the stabilizer subgroups $\left(\mathcal{I}\left(\Sigma_{g}\right)\right)_{\gamma}$ of simple closed curves $\gamma$. These stabilizer subgroups are supported on "simpler" subsurfaces, so we can use induction and Theorem 1.2 to analyze them.

Now, the condition $g \geq 1$ in Theorem 1.3 is necessary; indeed, for some partitions $P$ of the boundary components of $\Sigma_{0, n}$, there are no $P$-separating curves or $P$-bounding pairs. To find generators for the Torelli groups of genus 0 surfaces, we make one final definition. If $\left\{\gamma, \gamma^{\prime}\right\}$ is a pair of simple closed curves in $\Sigma_{g, n}$ whose geometric intersection number is 2 and whose algebraic intersection number is 0 (for instance, the pair $\left\{\gamma_{4}, \gamma_{5}\right\}$ in Figure 1.c), then it is easy to see that for any partition $P$ of the boundary components of $\Sigma_{g, n}$ we have $\left[T_{\gamma}, T_{\gamma^{\prime}}\right] \in \mathcal{I}\left(\Sigma_{g, n}, P\right)$. We will call these commutators of simply intersecting pairs. Our final theorem is the following:

Theorem 1.5 For any genus 0 partitioned surface $\left(\Sigma_{0, n}, P\right)$, the group $\mathcal{I}\left(\Sigma_{0, n}, P\right)$ is generated by twists about $P$-separating curves, twists about $P$-bounding pairs, and commutators of simply intersecting pairs.

History and comments At least two special cases of our construction have appeared in the literature. The simplest appears in the work of Hain [8], who (in our notation) considered $\mathcal{I}\left(\Sigma_{g, n}, P\right)$ with $P=\{\{1\}, \ldots,\{n\}\}$, which he defined as the subgroup of $\mathcal{I}\left(\Sigma_{g}\right)$ fixing $n$ discs. This is the "largest" possible definition of the Torelli group, and is rather easily related to the closed surface case. However, it does not have good functoriality properties, and it seems difficult to use it in inductive proofs (though, as Hain notes, it does have interesting interpretations in terms of algebraic geometry).

The other special case appears in the work of Johnson [15] and van den Berg [20]. In our notation, Johnson considered $\mathcal{I}\left(\Sigma_{g, n}, P\right)$ with $P=\{\{1, \ldots, n\}\}$. This is the "smallest" possible definition of the Torelli group, and it is functorial under embeddings. However, it is not closed under restriction except in the simplest possible cases, and great care has to be exercised when using it in inductive proofs. This work was continued in the unpublished thesis of van den Berg [20], who indicated how to extend Johnson's calculation of $\mathrm{H}_{1}\left(\mathcal{I}\left(\Sigma_{g}\right) ; \mathbb{Z}\right)$ to surfaces with boundary using Johnson's definition of the Torelli group on surfaces with boundary. She also gave a very brief sketch of the 
identification of the kernel of the exact sequence in Theorem 1.2 for the cases she was considering, though she did not prove that the associated map was surjective.

The history of Birman and Powell's result is rather interesting. Though it is a fundamental result used in nearly every subsequent paper on the Torelli group, their proof is the only one we are aware of in the literature. The story begins with a paper of Klingen [17] in which he gave an algorithm for computing a presentation of $\mathrm{Sp}_{2 g}(\mathbb{Z})$. In Birman's paper [3], she followed this algorithm. Assembling results from a paper of Magnus [18] and the unpublished thesis of Gold [6], she showed that Klingen's presentation has 5 families of generators and 67 families of relations. After an absolutely heroic calculation (whose details, needless to say, are only briefly sketched in Birman's short paper), she reduced this to a presentation with 3 families of generators and 10 families of relations.

These 3 families of generators are the images of a standard set of generators for $\operatorname{Mod}\left(\Sigma_{g}\right)$. She calculated that all but a few of these families of relations lift to relations in $\operatorname{Mod}\left(\Sigma_{g}\right)$. The relations in $\operatorname{Sp}_{2 g}(\mathbb{Z})$ which do not lift to relations in $\operatorname{Mod}\left(\Sigma_{g}\right)$ lift to normal generators for $\mathcal{I}\left(\Sigma_{g}\right)$. In Powell's paper, he showed how to express these normal generators for $\mathcal{I}\left(\Sigma_{g}\right)$ as products of twists about separating curves and bounding pairs, thus establishing the result.

Our proof seems to be the first to appear in the literature which does not depend on unpublished results and for which full details are given. Of course, $K$-theoretic methods have by now yielded simpler presentations of $\mathrm{Sp}_{2 n}(\mathbb{Z})$ than Klingen's presentation (see, eg, [7, Theorem 9.2.13]), but these constructions are quite involved, and it is nontrivial to perform Birman and Powell's analysis on them to get generators for the Torelli group. Our method has two advantages over such an approach. First, our methods are "intrinsic" to the theory of mapping class groups. Second, for the most part we avoid complicated group-theoretic calculations.

Acknowledgements I wish to thank my advisor Benson Farb for introducing me to the Torelli group and commenting extensively on previous incarnations of this paper, Ben Wieland for several useful discussions, and Justin Malestein for many useful discussions and corrections (in particular, Justin observed Lemma 6.7, which significantly simplified my original argument). Additionally, I wish to thank Dan Margalit and Matt Day for some corrections and Yair Minsky for allowing me to use his drawing of the Farey graph. Finally, I wish to thank the mathematics department of the Georgia Institute of Technology for their hospitality during the time in which part of this paper was conceived.

Outline We begin with two sections outlining preliminaries. Next, we define the Torelli group on a surface with boundary in Section 3.1 and discuss morphisms between 
different Torelli groups in Section 3.2. After a discussion of how to restrict Torelli groups to subsurfaces in Section 3.3, we prove our analogue of the Birman exact sequence in Section 4. In Section 5 we find generators for the Torelli groups, proving Theorems 1.3 and 1.5. The proofs of these theorems depend on the simple-connectedness of $\mathcal{C}\left(\Sigma_{g}\right) / \mathcal{I}\left(\Sigma_{g}\right)$ for $g \geq 2$, which is proven in Section 6 (see the beginning of that section for an outline of this lengthy proof). We conclude with an Appendix in which we prove several useful lemmas about the topology of surfaces.

Throughout this paper, all homology groups will have $\mathbb{Z}$-coefficients. A summand $A$ of a module $M$ is a submodule so that there exists another submodule $B$ with $M=A \oplus B$. We will often abuse notation and discuss the span $\left\langle X_{1}, \ldots, X_{k}\right\rangle$ of a set of submodules of a single module $X$. The algebraic intersection of two homology classes $h_{1}, h_{2}$ will be denoted by $i_{a}\left(h_{1}, h_{2}\right)$. The geometric intersection number of two simple closed curves $\gamma_{1}, \gamma_{2}$ will be denoted by $i_{g}\left(\gamma_{1}, \gamma_{2}\right)$. Finally, the flag complex with vertices $X$ and adjacency relation $R$ is the simplicial complex whose simplices are sets $\left\{x_{1}, \ldots, x_{k}\right\} \subset X$ so that $x_{i} R x_{j}$ for all $i$ and $j$.

\section{Preliminaries}

\subsection{The Birman exact sequences}

In this section, we will give a detailed review of the exact sequences of Birman and Johnson $[2 ; 4 ; 14]$ which describe the effect on the mapping class group of gluing a disc to a boundary component. We will need the following definition:

Definition Consider a surface $\Sigma_{g, n}$. Let $x \in \Sigma_{g, n}$ be a point. We define the mapping class group relative to $x, \operatorname{Mod}\left(\Sigma_{g, n}, x\right)$, to be the group of orientation-preserving homeomorphisms which fix $x$ and the boundary modulo isotopies fixing $x$ and the boundary.

Let $b$ be a boundary component of $\Sigma_{g, n}$. There is a natural embedding $\Sigma_{g, n} \hookrightarrow \Sigma_{g, n-1}$ induced by gluing a disc to $b$. Let $x$ be a point in the interior of the new disc. Clearly we can factor the induced map $\operatorname{Mod}\left(\Sigma_{g, n}\right) \rightarrow \operatorname{Mod}\left(\Sigma_{g, n-1}\right)$ into a composition

$$
\operatorname{Mod}\left(\Sigma_{g, n}\right) \longrightarrow \operatorname{Mod}\left(\Sigma_{g, n-1}, x\right) \longrightarrow \operatorname{Mod}\left(\Sigma_{g, n-1}\right) .
$$

Now let $U \Sigma_{g, n-1}$ be the unit tangent bundle of $\Sigma_{g, n-1}$ and $\tilde{x}$ be any lift of $x$ to $U \Sigma_{g, n-1}$. The combined work of Birman [2] and Johnson [14] shows that (except for the degenerate case $(g, n)=(1,1))$ all of our groups fit into the following commutative diagram with exact rows and columns: 

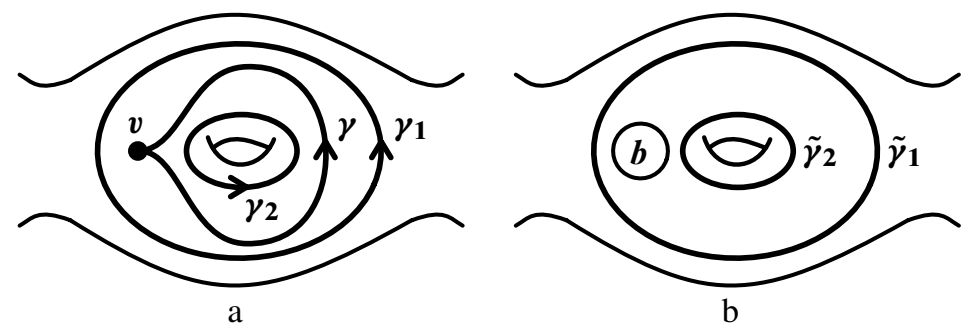

Figure 2: a: Image of a simple closed curve in $\operatorname{Mod}\left(\Sigma_{g, n-1}, x\right)$

b: Lift of a simple closed curve to $\operatorname{Mod}\left(\Sigma_{g, n}\right)$

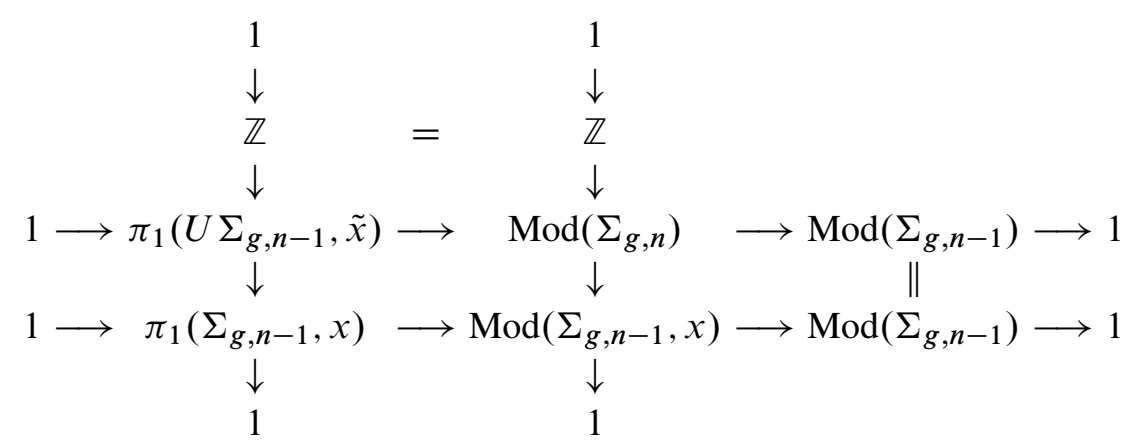

The $\mathbb{Z}$ in the first column is the loop in the fiber, while the $\mathbb{Z}$ in the second column corresponds to the Dehn twist about the filled-in boundary component.

For $\gamma \in \pi_{1}\left(\Sigma_{g, n-1}, x\right)$, let $\sigma_{\gamma}$ be the element of $\operatorname{Mod}\left(\Sigma_{g, n-1}, x\right)$ associated to $\gamma$ (hence $\sigma_{\gamma}$ is the mapping class which "pushes $x$ around $\gamma$ "). If $\gamma$ is a simple closed curve, then there is a nice formula for $\sigma_{\gamma}$ (see Figure 2.a). Namely, let $\gamma_{1}$ and $\gamma_{2}$ be the boundary components of a regular neighborhood of $\gamma$. The orientation of $\gamma$ induces orientations on $\gamma_{1}$ and $\gamma_{2}$; assume that $\gamma$ lies to the left of $\gamma_{1}$ and to the right of $\gamma_{2}$. Then $\sigma_{\gamma}=T_{\gamma_{1}} T_{\gamma_{2}}^{-1}$.

Continue to assume that $\gamma$ is a simple closed curve. We will construct a natural lift $\tilde{\sigma}_{\gamma}$ of $\sigma_{\gamma}$ to $\pi_{1}\left(U \Sigma_{g, n-1}, \tilde{x}\right) \subset \operatorname{Mod}\left(\Sigma_{g, n}\right)$ (we reiterate that our construction depends on the simplicity of $\gamma$ ). Recall that we have been considering $\Sigma_{g, n-1}$ to be $\Sigma_{g, n}$ with a disc glued to $b$. In the other direction, we can consider $\Sigma_{g, n}$ to be $\Sigma_{g, n-1}$ with the point $x$ blown up to a boundary component. Two such identifications may differ by a power of $T_{b}$; however, since $T_{b}$ fixes both $\gamma_{1}$ and $\gamma_{2}$, there are well-defined lifts $\tilde{\gamma}_{1}$ and $\tilde{\gamma}_{2}$ of the $\gamma_{i}$ to $\Sigma_{g, n}$ (see Figure 2.b). It is not hard to see that

$$
\tilde{\sigma}_{\gamma}:=T_{\tilde{\gamma}_{1}} T_{\tilde{\gamma}_{2}}^{-1}
$$

is a lift of $\sigma_{\gamma}$. 
Remark While the map

$$
\sigma .: \pi_{1}\left(\Sigma_{g, n-1}, x\right) \longrightarrow \operatorname{Mod}\left(\Sigma_{g, n-1}, x\right)
$$

is a homomorphism, the map $\tilde{\sigma}$. (which is only defined on simple closed curves) does not extend to a homomorphism.

\subsection{Groups acting on simplicial complexes}

In this section, we will prove a theorem (really, a corollary of a theorem of Armstrong [1]) which we will use to find generating sets for the Torelli group. We will need the following definition:

Definition A group $G$ acts on a simplicial complex $X$ without rotations if for all simplices $s$ of $X$ the stabilizer $G_{s}$ stabilizes $s$ pointwise.

The following is our theorem:

Theorem 2.1 Let $G$ act without rotations on a simply-connected simplicial complex $X$. Then $G$ is generated by the set

$$
\bigcup_{v \in X^{(0)}} G_{v}
$$

if and only if $X / G$ is simply-connected.

Proof Let $H$ be the subgroup of $G$ generated by the indicated set (note that $H$ is normal). Observe that since $G$ acts without rotations, we can subdivide $X$ without changing $H$. After possibly subdividing twice, the work of Armstrong [1] implies that there is an exact sequence

$$
1 \longrightarrow H \longrightarrow G \longrightarrow \pi_{1}(X / G) \longrightarrow 1 .
$$

This clearly implies the theorem. We briefly indicate the construction of the exact sequence. Let $\pi: X \rightarrow X / G$ be the projection, and fix a vertex $v$ of $X$. We define a homomorphism $j: G \rightarrow \pi_{1}(X / G, \pi(v))$ in the following way. For $g \in G$, let $\ell$ be a path in $X$ from $v$ to $g v$. Then $j(g):=\pi \circ \ell$ is a loop in $X / G$ based at $\pi(v)$. Since $X$ is simply-connected, $j$ is well-defined, and it is clear that $j$ is a surjective homomorphism. Now, if $g w=w$ for a vertex $w \in X$, we claim that $j(g)=1$. Indeed, let $\ell^{\prime}$ be a path from $v$ to $w$. Then $\ell^{\prime} \cdot\left(g\left(\ell^{\prime}\right)\right)^{-1}$ is a path from $v$ to $g v$ which clearly projects to a null-homotopic loop in $X / G$, proving the claim. The bulk of Armstrong's work, therefore, consists of showing that if $g \in \operatorname{ker}(j)$, then $g \in H$. We refer the reader to Armstrong's paper [1, pages 643-645] for the details. 


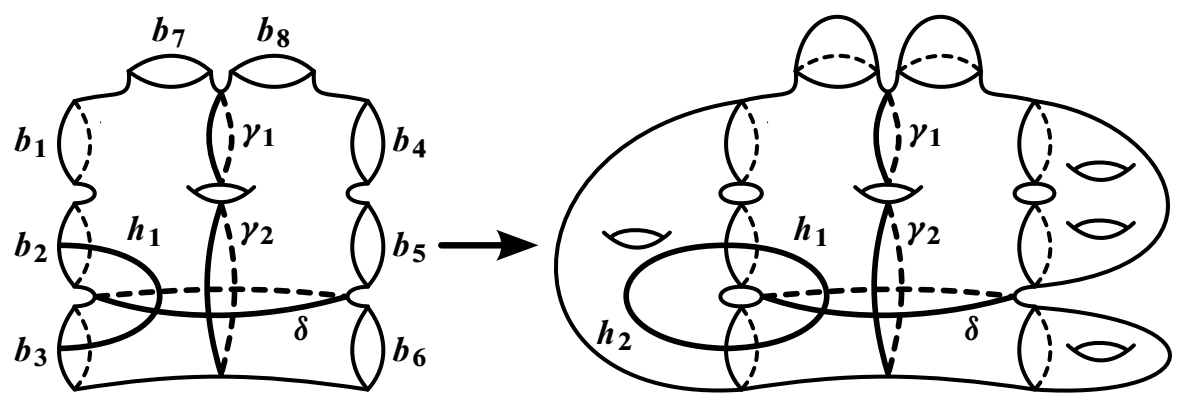

Figure 3: A capping of $\left(\Sigma_{1,8},\{\{1,2,3\},\{4,5\},\{6\},\{7\},\{8\}\}\right)$

\section{Definition of the Torelli group on a surface with boundary}

\subsection{Definition}

In this section, we will define the Torelli group on a surface with boundary. As discussed in the introduction, our main goal is to understand the subgroups of $\operatorname{Mod}(\Sigma)$ which arise as $i_{*}^{-1}\left(\mathcal{I}\left(\Sigma_{g}\right)\right)$ for embeddings $i: \Sigma \hookrightarrow \Sigma_{g}$. We observed there that different embeddings yield different "Torelli groups" for $\Sigma$.

Recall that in the introduction we indicated that to distinguish these different Torelli groups we would attach to a surface $\Sigma$ a partition $P$ of its boundary components; the pair $(\Sigma, P)$ will be called a partitioned surface. Associated to an embedding $i: \Sigma \hookrightarrow \Sigma_{g}$ is a partition

$$
P=\left\{\partial S \mid S \text { is a component of } \Sigma_{g} \backslash \operatorname{Int}(\Sigma)\right\}
$$

of the boundary components of $\Sigma$. The following is a useful shorthand for the embeddings which give rise to a partition $P$ :

Definition A capping of a partitioned surface $(\Sigma, P)$ (see Figure 3$)$ is an embedding

$$
\Sigma \hookrightarrow \Sigma_{g}
$$

so that for each component $S$ of $\Sigma_{g} \backslash \operatorname{Int}(\Sigma)$, the set of boundary components of $S$ is exactly equal to an element of $P$.

The follow obvious lemma says that that every embedding is a capping for an appropriate partition:

Lemma 3.1 Let $i: \Sigma \hookrightarrow \Sigma_{g}$ be an embedding. Set

$$
P=\left\{\partial S \subset \partial \Sigma \mid S \text { is a component of } \Sigma_{g} \backslash \operatorname{Int}(\Sigma)\right\} .
$$


Then $i$ is a capping of $(\Sigma, P)$.

We now define the Torelli group of a partitioned surface.

Definition For a partitioned surface $(\Sigma, P)$, let $\mathcal{I}(\Sigma, P):=i_{*}^{-1}\left(\mathcal{I}\left(\Sigma_{g}\right)\right)$ for any capping $i: \Sigma \hookrightarrow \Sigma_{g}$.

Of course, it is not at all clear that this definition of $\mathcal{I}(\Sigma, P)$ is independent of the chosen capping. Also, it seems rather ad-hoc - one would like to say that $\mathcal{I}(\Sigma, P)$ is the subgroup of $\operatorname{Mod}(\Sigma)$ fixing some sort of homology group. We will answer these objections simultaneously by defining a certain intrinsic "homology group" $\mathrm{H}_{1}^{P}(\Sigma)$ and then proving Theorem 3.3, which says that $\mathcal{I}(\Sigma, P)$ is exactly the subgroup of $\operatorname{Mod}(\Sigma)$ acting trivially on $\mathrm{H}_{1}^{P}(\Sigma)$.

The construction of $\mathrm{H}_{1}^{P}(\Sigma)$ is a two step process. For the first step, observe that in Figure 3 the mapping class $T_{\gamma_{1}} T_{\gamma_{2}}^{-1}$ is an element of $\mathcal{I}\left(\Sigma_{8}\right)$, but it does not fix $\mathrm{H}_{1}\left(\Sigma_{1,8}\right)$. The problem is that (picking appropriate orientations for everything)

$$
\left[\gamma_{1}\right]-\left[\gamma_{2}\right]=\left[b_{1}\right]+\left[b_{2}\right]+\left[b_{3}\right]+\left[b_{7}\right] \neq 0 .
$$

This motivates the following definition:

Definition Consider a partitioned surface $(\Sigma, P)$, and enumerate the partition $P$ :

$$
P=\left\{\left\{b_{1}^{1}, \ldots, b_{k_{1}}^{1}\right\}, \ldots,\left\{b_{1}^{m}, \ldots, b_{k_{m}}^{m}\right\}\right\} .
$$

Orient the boundary components $b_{i}^{j}$ so that $\sum_{i, j}\left[b_{i}^{j}\right]=0$ in $\mathrm{H}_{1}(\Sigma)$. Define

$$
\begin{aligned}
\partial \mathrm{H}_{1}^{P}(\Sigma) & =\left\langle\left[b_{1}^{1}\right]+\ldots+\left[b_{k_{1}}^{1}\right], \ldots,\left[b_{1}^{m}\right]+\ldots+\left[b_{k_{m}}^{m}\right]\right\rangle \subset \mathrm{H}_{1}(\Sigma), \\
\overline{\mathrm{H}}_{1}^{P}(\Sigma) & =\mathrm{H}_{1}(\Sigma) / \partial \mathrm{H}_{1}^{P}(\Sigma) .
\end{aligned}
$$

The following lemma is immediate from the definitions:

Lemma 3.2 If $i: \Sigma \hookrightarrow \Sigma_{g}$ is a capping of a partitioned surface $(\Sigma, P)$, then there is an induced injection $i_{*}: \overline{\mathrm{H}}_{1}^{P}(\Sigma) \hookrightarrow \mathrm{H}_{1}\left(\Sigma_{g}\right)$.

For the second step of the construction of $\mathrm{H}_{1}^{P}(\Sigma)$, observe that in Figure 3 the mapping class $T_{\delta}$ does not fix the homology class $\left[h_{1}\right]+\left[h_{2}\right]$ (where the $h_{i}$ are the indicated arcs and $\left[h_{i}\right]$ is the chain corresponding to $h_{i}$; only the sum $\left[h_{1}\right]+\left[h_{2}\right]$ is a cycle), and hence is not in $\mathcal{I}\left(\Sigma_{8}\right)$. However, it does fix $\overline{\mathrm{H}}_{1}^{P}(\Sigma)$. The difficulty is that we really need elements of $\mathcal{I}\left(\Sigma_{1,8},\{\{1,2,3\},\{4,5\},\{6\},\{7\},\{8\}\}\right)$ to fix $\left[h_{1}\right]$, which is a homology class relative to points on the boundary components. This motivates the following definition: 
Definition Fix a partitioned surface $(\Sigma, P)$, and let $Q$ be a set containing one point from each boundary component of $\Sigma$. Define $\mathrm{H}_{1}^{P}(\Sigma)$ to equal the image of the following submodule of $\mathrm{H}_{1}(\Sigma, Q)$ in $\mathrm{H}_{1}(\Sigma, Q) / \partial \mathrm{H}_{1}^{P}(\Sigma)$ :

$$
\begin{aligned}
\left\langle\left\{[h] \in \mathrm{H}_{1}(\Sigma, Q) \mid\right.\right. & \text { either } h \text { is a simple closed curve or } h \text { is a properly } \\
& \text { embedded arc from } q_{1} \text { to } q_{2} \text { with } q_{1}, q_{2} \in Q \text { lying } \\
& \text { in boundary components } b_{1} \text { and } b_{2} \text { with }\left\{b_{1}, b_{2}\right\} \subset p \\
& \text { for some } p \in P\}\rangle
\end{aligned}
$$

We remark that in the future we will omit mention of $Q$ and instead refer to the homology classes of arcs between boundary components, the set $Q$ being understood. If boundary components $b_{1}$ and $b_{2}$ satisfy $b_{1}, b_{2} \in p$ for some $p \in P$, we will call them $P$-adjacent boundary components.

Remark The idea of using relative homology classes to analyze the Torelli groups on surfaces with boundary is due to Johnson [15].

It is clear that $\operatorname{Mod}(\Sigma)$ acts upon $\mathrm{H}_{1}^{P}(\Sigma)$. We now prove the following:

Theorem 3.3 For a partitioned surface $(\Sigma, P)$, the group $\mathcal{I}(\Sigma, P)$ is exactly the subgroup of $\operatorname{Mod}(\Sigma)$ which acts trivially on $\mathrm{H}_{1}^{P}(\Sigma)$. In particular, $\mathcal{I}(\Sigma, P)$ is welldefined.

Proof Fix a capping $i: \Sigma \hookrightarrow \Sigma_{g}$ of $(\Sigma, P)$. Define the following subsets of $\mathrm{H}_{1}\left(\Sigma_{g}\right)$ :

$$
\begin{aligned}
Q_{1}=\left\{[h] \in \mathrm{H}_{1}\left(\Sigma_{g}\right) \mid\right. & \left.h \text { is a simple closed curve in } \Sigma_{g} \backslash \Sigma\right\} \\
Q_{2}=\left\{[h] \in \mathrm{H}_{1}\left(\Sigma_{g}\right) \mid\right. & h \text { is a simple closed curve in } \Sigma\} \\
Q_{3}=\left\{\left[h_{1}\right]+\left[h_{2}\right] \in \mathrm{H}_{1}\left(\Sigma_{g}\right) \mid\right. & h_{1} \text { is a properly embedded arc in } \Sigma \text { between } \\
& P \text {-adjacent boundary components and } h_{2} \text { is } \\
& \text { a properly embedded arc in } \Sigma_{g} \backslash \operatorname{Int}(\Sigma) \\
& \text { with the same endpoints as } \left.h_{1}\right\}
\end{aligned}
$$

For an example of an element of $Q_{3}$, see Figure 3. It is clear that

$$
\left\langle Q_{1} \cup Q_{2} \cup Q_{3}\right\rangle=\mathrm{H}_{1}\left(\Sigma_{g}\right) .
$$

For $f \in \operatorname{Mod}(\Sigma)$, the mapping class $i_{*}(f)$ fixes every element of $Q_{1}$. Also, by Lemma 3.2, the mapping class $i_{*}(f)$ fixes $[h] \in Q_{2}$ if and only if $f$ fixes the corresponding element of $\mathrm{H}_{1}^{P}(\Sigma)$. Finally, we claim that $i_{*}(f)$ fixes $\left[h_{1}\right]+\left[h_{2}\right] \in Q_{3}$ if and only if $f$ fixes $\left[h_{1}\right] \in \mathrm{H}_{1}^{P}(\Sigma)$. Indeed, the reverse implication is trivial, while for the forward 
implication, observe that if $f$ does not fix $\left[h_{1}\right] \in \mathrm{H}_{1}^{P}(\Sigma)$, then $\left[h_{1}\right]-f\left(\left[h_{1}\right]\right) \in \overline{\mathrm{H}}_{1}^{P}(\Sigma)$ is nonzero, so by Lemma 3.2 we have

$$
\left(\left[h_{1}\right]+\left[h_{2}\right]\right)-i_{*}(f)\left(\left[h_{1}\right]+\left[h_{2}\right]\right)=i\left(\left[h_{1}\right]-f\left(\left[h_{1}\right]\right)\right) \neq 0 ;
$$

ie, $i_{*}(f)$ does not fix $\left[h_{1}\right]+\left[h_{2}\right]$. We conclude that $i_{*}(f)$ acts trivially on $\mathrm{H}_{1}\left(\Sigma_{g}\right)$ if and only if $f$ acts trivially on $\mathrm{H}_{1}^{P}(\Sigma)$, as desired.

We now prove the following lemma, which describes the natural "algebraic intersection pairing" on $\mathrm{H}_{1}^{P}(\Sigma)$ :

Lemma 3.4 Fix a partitioned surface $(\Sigma, P)$, and let $Q \subset \partial \Sigma$ be the set from the definition of $\mathrm{H}_{1}^{P}(\Sigma)$. Then the algebraic intersection pairing $i_{a}(\cdot, \cdot)$ on $\mathrm{H}_{1}(\Sigma, Q)$ induces a pairing on $\mathrm{H}_{1}^{P}(\Sigma)$ (which we will continue to call $i_{a}(\cdot, \cdot)$ ). This pairing is preserved by $\operatorname{Mod}(\Sigma)$, and for a simple closed curve $\gamma$, the mapping class $T_{\gamma}$ acts upon $\mathrm{H}_{1}^{P}(\Sigma)$ by the transvection

$$
h \longmapsto h+i_{a}(h,[\gamma])[\gamma] .
$$

Proof The only nontrivial part of this lemma is that $i_{a}$ is well defined on $\mathrm{H}_{1}^{P}(\Sigma)$. Let $H \subset \mathrm{H}_{1}(\Sigma, Q)$ be the pull-back of $\mathrm{H}_{1}^{P}(\Sigma)$. We must show that for $b \in \partial \mathrm{H}_{1}^{P}(\Sigma)$, the map $i_{a}(b, \cdot)$ restricts to the 0 map on $H$. Enumerating the partition $P$ as

$$
P=\left\{\left\{b_{1}^{1}, \ldots, b_{k_{1}}^{1}\right\}, \ldots,\left\{b_{1}^{m}, \ldots, b_{k_{m}}^{m}\right\}\right\},
$$

we can assume that $b$ is one of the generators $\left[b_{1}^{i}\right]+\cdots+\left[b_{k_{i}}^{i}\right]$ of $\partial \mathrm{H}_{1}^{P}(\Sigma)$. If $h$ is a simple closed curve or an arc between boundary components $b_{r}^{j}$ and $b_{s}^{j}$ with $i \neq j$, then trivially $i_{a}(b,[h])=0$. If instead $h$ is an arc between boundary components $b_{r}^{i}$ and $b_{s}^{i}$, then we have $i_{a}(b,[h])=i_{a}\left(\left[b_{r}^{j}\right],[h]\right)+i_{a}\left(\left[b_{s}^{j}\right],[h]\right)=0$. Since $i_{a}(b, \cdot)$ vanishes on generators for $H$, it vanishes on $H$, as desired.

We conclude this section by discussing typical elements of $\mathcal{I}(\Sigma, P)$.

Definition Fix a partitioned surface $(\Sigma, P)$.

- A $P$-separating curve is a simple closed curve $\gamma$ so that $[\gamma]=0$ in $\mathrm{H}_{1}^{P}(\Sigma)$. Equivalently, $\gamma$ is a separating curve and for any boundary components $b_{1}$ and $b_{2}$ with $b_{1}, b_{2} \in p$ for some $p \in P$, the curve $\gamma$ does not separate $b_{1}$ from $b_{2}$.

- A twist about a $P$-bounding pair equals $T_{\gamma_{1}} T_{\gamma_{2}}^{-1}$ for disjoint, nonisotopic simple closed curves $\gamma_{1}$ and $\gamma_{2}$ so that for some choice of orientations we have $\left[\gamma_{1}\right]=\left[\gamma_{2}\right]$ (as elements of $\left.\mathrm{H}_{1}^{P}(\Sigma)\right)$. 
These are all elements of $\mathcal{I}(\Sigma, P)$ :

Lemma 3.5 Fix a partitioned surface $(\Sigma, P)$, and let $f \in \operatorname{Mod}(\Sigma)$ be a twist about either a separating curve or a bounding pair. Then $f \in \mathcal{I}(\Sigma, P)$ if and only if $f$ is a twist about a $P$-separating curve or a $P$-bounding pair.

Proof The $P$-separating curves and $P$-bounding pairs are exactly the separating curves and bounding pairs in $\Sigma$ which remain separating curves and bounding pairs in any capping of $(\Sigma, P)$.

\subsection{Morphisms between Torelli groups}

In this section, we construct a category TSur whose objects are partitioned surfaces $(\Sigma, P)$ and whose morphisms from $\left(\Sigma_{g_{1}, n_{1}}, P_{1}\right)$ to $\left(\Sigma_{g_{2}, n_{2}}, P_{2}\right)$ are exactly those embeddings $i: \Sigma_{g_{1}, n_{1}} \hookrightarrow \Sigma_{g_{2}, n_{2}}$ which induce morphisms $i_{*}: \mathcal{I}\left(\Sigma_{g_{1}, n_{1}}, P_{1}\right) \rightarrow$ $\mathcal{I}\left(\Sigma_{g_{2}, n_{2}}, P_{2}\right)$. There is one obvious condition on such embeddings : for any $P_{1}-$ separating curve $\gamma$, the curve $i(\gamma)$ must be a $P_{2}$-separating curve. To translate this into a condition on the partitions, we need some notation. Let $S$ be any component of $\Sigma_{g_{2}, n_{2}} \backslash \operatorname{Int}\left(\Sigma_{g_{1}, n_{1}}\right)$. Observe that $S$ may in fact consist of a single boundary component of $\Sigma_{g_{1}, n_{1}}$ which is also a boundary component of $\Sigma_{g_{2}, n_{2}}$. Let $B_{S}$ be the boundary components of $\Sigma_{g_{2}, n_{2}}$ which lie in $S$, and let $B_{S}^{\prime}$ be the boundary components of $\Sigma_{g_{1}, n_{1}}$ which lie in $S$. Our category is the following:

Definition The Torelli surface category (denoted TSur) is the category whose objects are partitioned surfaces $(\Sigma, P)$ and whose morphisms from $\left(\Sigma_{g_{1}, n_{1}}, P_{1}\right)$ to ( $\Sigma_{g_{2}, n_{2}}, P_{2}$ ) are embeddings $\Sigma_{g_{1}, n_{1}} \hookrightarrow \Sigma_{g_{2}, n_{2}}$ satisfying the following two conditions:

(1) Each set $B_{S}^{\prime}$ is contained in some $p \in P_{1}$.

(2) Consider $b_{1}, b_{2} \in p$ for some $p \in P_{2}$. Assume that $b_{1} \in B_{S}$ for some $S$ and that $b_{2} \in B_{S^{\prime}}$ for some $S^{\prime} \neq S$. Then $B_{S}^{\prime} \cup B_{S^{\prime}}^{\prime} \subset q$ for some $q \in P_{1}$. Less formally, there is a well-defined "retraction map" $P_{2} \rightarrow P_{1}$.

Condition 1 is necessary for all $P_{1}$-separating curves in $\Sigma_{g_{1}, n_{1}}$ to remain separating curves in $\Sigma_{g_{2}, n_{2}}$, and condition 2 is necessary to assure that they in fact are $P_{2}-$ separating curves. The following theorem says that these are exactly the morphisms we want:

Theorem 3.6 Let $\left(\Sigma_{g_{1}, n_{1}}, P_{1}\right)$ and $\left(\Sigma_{g_{2}, n_{2}}, P_{2}\right)$ be partitioned surfaces. Fix an embedding $i: \Sigma_{g_{1}, n_{1}} \hookrightarrow \Sigma_{g_{2}, n_{2}}$. Then

$$
i_{*}\left(\mathcal{I}\left(\Sigma_{g_{1}, n_{1}}, P_{1}\right)\right) \subset \mathcal{I}\left(\Sigma_{g_{2}, n_{2}}, P_{2}\right)
$$

if and only if $i$ is a morphism of TSur. 


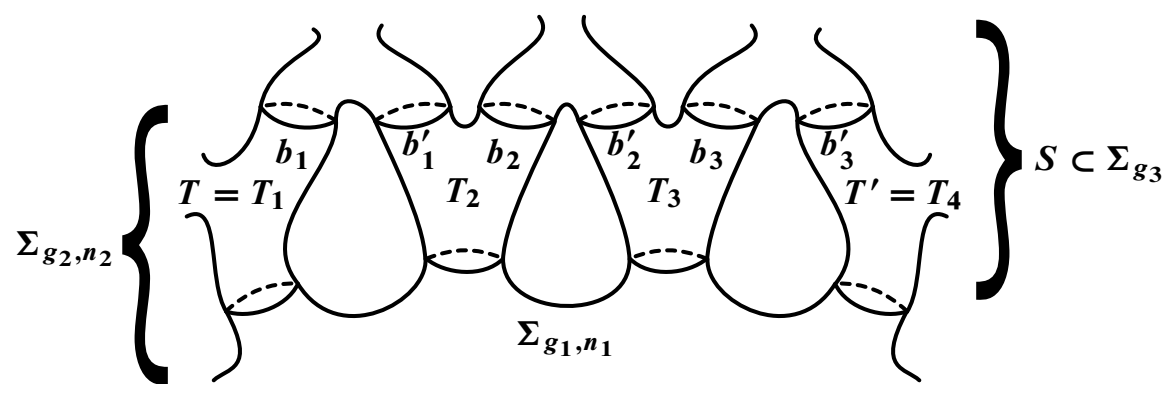

Figure 4: Portion of a component of $\Sigma_{g_{3}} \backslash \operatorname{Int}\left(\Sigma_{g_{1}, n_{1}}\right)$

Corollary 3.7 $\mathcal{I}$ is a functor from TSur to the category of groups and homomorphisms.

We begin by proving a special case.

Proof of reverse implication of Theorem 3.6 when $\boldsymbol{n}_{2}=\mathbf{0}$ In this case, condition 2 is vacuous. By Lemma 3.1, there is a partition $P_{1}^{\prime}$ of the boundary components of $\Sigma_{g_{1}, n_{1}}$ so that $i: \Sigma_{g_{1}, n_{1}} \hookrightarrow \Sigma_{g_{2}}$ is a capping of $\left(\Sigma_{g_{1}, n_{1}}, P_{1}^{\prime}\right)$. Observe that condition 1 says that for all $p^{\prime} \in P_{1}^{\prime}$, there is some $p \in P_{1}$ with $p^{\prime} \subset p$. Defining $H \subset \mathrm{H}_{1}^{P_{1}}(\Sigma)$ to equal

$\langle\{[h] \mid h$ is a simple closed curve or a properly embedded arc between $P_{1}^{\prime}$-adjacent boundary components $\left.\}\right\rangle$,

we conclude that $H$ surjects onto $\mathrm{H}_{1}^{P_{1}^{\prime}}(\Sigma)$. Theorem 3.3 therefore implies that

$$
\mathcal{I}\left(\Sigma_{g_{1}, n_{1}}, P_{1}\right) \subset \mathcal{I}\left(\Sigma_{g_{1}, n_{1}}, P_{1}^{\prime}\right)=i_{*}^{-1}\left(\mathcal{I}\left(\Sigma_{g_{2}}\right)\right),
$$

whence the theorem.

We now prove the general case.

Proof of Theorem 3.6 in the general case As was mentioned before the theorem, if condition 1 is not satisfied, then there is some $P_{1}$-separating curve $\gamma$ in $\Sigma_{g_{1}, n_{1}}$ so that $i(\gamma)$ is not a separating curve, and if condition 2 is not satisfied, then there is some $P_{1}$-separating curve $\gamma$ in $\Sigma_{g_{1}, n_{1}}$ so that $i(\gamma)$ is not a $P_{2}$-separating curve. Lemma 3.5 therefore implies the necessity of the 2 conditions. We must prove their sufficiency.

Let $j: \Sigma_{g_{2}, n_{2}} \hookrightarrow \Sigma_{g_{3}}$ be a capping of $\left(\Sigma_{g_{2}, n_{2}}, P_{2}\right)$. We claim that $j \circ i: \Sigma_{g_{1}, n_{1}} \hookrightarrow \Sigma_{g_{3}}$ satisfies the conditions of the theorem (for the partition $P_{1}$ of the boundary components 
of $\left.\Sigma_{g_{1}, n_{1}}\right)$. Indeed, if $S$ is a component of $\Sigma_{g_{3}} \backslash \operatorname{Int}\left(\Sigma_{g_{1}, n_{1}}\right)$, then $B_{S}^{\prime}$ is a union of $B_{T}^{\prime}$ for certain components $T$ of $\Sigma_{g_{2}, n_{2}} \backslash \operatorname{Int}\left(\Sigma_{g_{1}, n_{1}}\right)$. By condition 1, each $B_{T}^{\prime}$ is contained in some $p \in P_{1}$. It is not hard to see from the definition of a capping (see Figure 4) that if $T$ and $T^{\prime}$ are 2 components of $\Sigma_{g_{2}, n_{2}} \backslash \operatorname{Int}\left(\Sigma_{g_{1}, n_{1}}\right)$ so that $B_{T}^{\prime} \cup B_{T^{\prime}}^{\prime} \subset$ $B_{S}^{\prime}$, then there are components $T=T_{1}, \ldots, T_{k}=T^{\prime}$ of $\Sigma_{g_{2}, n_{2}} \backslash \operatorname{Int}\left(\Sigma_{g_{1}, n_{1}}\right)$ so that for $1 \leq i<k$ there is some $b_{i} \in B_{T_{i}}$ and $b_{i}^{\prime} \in B_{T_{i+1}}$ so that $b_{i}, b_{i}^{\prime} \in q$ for some $q \in P_{2}$. By condition 2, for $i=1, \ldots, k-1$ we have that $B_{T_{i}}^{\prime} \cup B_{T_{i+1}}^{\prime}$ is a subset of a single partition element of $P_{1}$. We conclude that $B_{T}^{\prime}$ and $B_{T^{\prime}}^{\prime}$ are contained in the same partition element of $P_{1}$. This implies that condition 1 holds. Since condition 2 is vacuous, this implies that $j \circ i$ does indeed satisfy the conditions of the theorem.

The special case of the theorem proven above therefore implies that

$$
j_{*} \circ i_{*}\left(\mathcal{I}\left(\Sigma_{g_{1}, n_{1}}, P_{1}\right)\right) \subset \mathcal{I}\left(\Sigma_{g_{3}}\right) .
$$

Since $\mathcal{I}\left(\Sigma_{g_{2}, n_{2}}, P_{2}\right)=j_{*}^{-1}\left(\mathcal{I}\left(\Sigma_{g_{3}}\right)\right)$, we conclude that

$$
i_{*}\left(\mathcal{I}\left(\Sigma_{g_{1}, n_{1}}, P_{1}\right)\right) \subset \mathcal{I}\left(\Sigma_{g_{2}, n_{2}}, P_{2}\right),
$$

as desired.

\subsection{Restriction properties of $\mathcal{I}$}

We now prove the following generalization of Theorem 3.3:

Theorem 3.8 Let $i: \Sigma_{g_{1}, n_{1}} \hookrightarrow \Sigma_{g_{2}, n_{2}}$ be any embedding, and let $P_{2}$ be any partition of the boundary components of $\Sigma_{g_{2}, n_{2}}$. There is then some partition $P_{1}$ of the boundary components of $\Sigma_{g_{1}, n_{1}}$ so that $\mathcal{I}\left(\Sigma_{g_{1}, n_{1}}, P_{1}\right)=i_{*}^{-1}\left(\mathcal{I}\left(\Sigma_{g_{2}, n_{2}}, P_{2}\right)\right.$.

Proof Let

$$
j: \Sigma_{g_{2}, n_{2}} \hookrightarrow \Sigma_{g_{3}}
$$

be a capping of $\left(\Sigma_{g_{2}, n_{2}}, P_{2}\right)$. By Lemma 3.1, there is some partition $P_{1}$ so that

$$
j \circ i: \Sigma_{g_{1}, n_{1}} \hookrightarrow \Sigma_{g_{3}}
$$

is a capping of $\left(\Sigma_{g_{1}, n_{1}}, P_{1}\right)$. It is not hard to show that

$$
i: \Sigma_{g_{1}, n_{1}} \hookrightarrow \Sigma_{g_{2}, n_{2}}
$$

satisfies the conditions of Theorem 3.6 (for the partitions $P_{i}$ of the boundary components of $\left.\Sigma_{g_{i}, n_{i}}\right)$, and hence $i_{*}$ induces a morphism from $\mathcal{I}\left(\Sigma_{g_{1}, n_{1}}, P_{1}\right)$ to $\mathcal{I}\left(\Sigma_{g_{2}, n_{2}}, P_{2}\right)$. The theorem is then an immediate corollary of Theorem 3.3 applied to both $j$ and $i \circ j$. 


\section{A Birman exact sequence for the Torelli groups}

In this section, we prove Theorem 1.2. Fix a partitioned surface $\left(\Sigma_{g, n}, P\right)$ with $n \geq 1$ and $(g, n) \neq 1$. Let $b$ be a boundary component of $\Sigma_{g, n}$, and let $p \in P$ be the subset containing $b$. Regarding $\Sigma_{g, n-1}$ as the surface which results from gluing a disc from $b$, let $P^{\prime}$ be the induced partition of the boundary components of $\Sigma_{g, n-1}$. The embedding $i: \Sigma_{g, n} \rightarrow \Sigma_{g, n-1}$ is clearly a morphism in TSur from $\left(\Sigma_{g, n}, P\right)$ to $\left(\Sigma_{g, n-1}, P^{\prime}\right)$, and hence there is an induced map $i_{*}: \mathcal{I}\left(\Sigma_{g, n}, P\right) \rightarrow \mathcal{I}\left(\Sigma_{g, n-1}, P^{\prime}\right)$. Now, choosing a point $x$ in the glued-in disc and a lift $\tilde{x}$ of $x$ to the unit tangent bundle $U \Sigma_{g, n-1}$, we discussed in Section 2.1 the following two exact sequences:

$$
\begin{aligned}
& 1 \longrightarrow \pi_{1}\left(U \Sigma_{g, n-1}, \tilde{x}\right) \longrightarrow \operatorname{Mod}\left(\Sigma_{g, n}\right) \longrightarrow \operatorname{Mod}\left(\Sigma_{g, n-1}\right) \longrightarrow 1, \\
& 1 \longrightarrow \mathbb{Z} \longrightarrow \pi_{1}\left(U \Sigma_{g, n-1}, \tilde{x}\right) \longrightarrow \pi_{1}\left(\Sigma_{g, n-1}, x\right) \longrightarrow 1 .
\end{aligned}
$$

Using exact sequence (1), we see that $\operatorname{ker}\left(i_{*}\right) \subset \pi_{1}\left(U \Sigma_{g, n-1}, \tilde{x}\right)$. However, it is rarely all of $\pi_{1}\left(U \Sigma_{g, n-1}, \tilde{x}\right)$ : for instance, unless $p=\{b\}$, the twist $T_{b}$ is not a twist about a $P$-separating curve, so it is not in the kernel. Theorem 1.2 says that $i_{*}$ is always surjective and also identifies its kernel. We will prove a slightly more precise version of Theorem 1.2. Before stating it, we need the following definition:

Definition Assume that a group $\Gamma$ splits as $G_{1} \oplus G_{2}$ and that $\phi: H_{1} \rightarrow G_{2}$ is a homomorphism, where $H_{1}$ is a subgroup of $G_{1}$. Then the graph of $\phi$ is the subgroup $\left\{(x, \phi(x)) \mid x \in H_{1}\right\}$ of $\Gamma$.

We will prove the following:

Theorem 4.1 With the notation as above, we have an exact sequence

$$
1 \longrightarrow K \longrightarrow \mathcal{I}\left(\Sigma_{g, n}, P\right) \longrightarrow \mathcal{I}\left(\Sigma_{g, n-1}, P^{\prime}\right) \longrightarrow 1
$$

with $K \subset \pi_{1}\left(U \Sigma_{g, n-1}, \tilde{x}\right)$ equal to the following:

- If $p=\{b\}$, then $K=\pi_{1}\left(U \Sigma_{g, n-1}, \tilde{x}\right)$.

- If $p \neq\{b\}$, then $\pi_{1}\left(U \Sigma_{g, n-1}, \tilde{x}\right)$ splits as $\pi_{1}\left(\Sigma_{g, n-1}, x\right) \oplus \mathbb{Z}$ and $K$ equals the graph of a homomorphism $\phi: K^{\prime} \rightarrow \mathbb{Z}$ for the kernel $K^{\prime} \subset \pi_{1}\left(\Sigma_{g, n-1}, x\right)$ of the natural map $\pi_{1}\left(\Sigma_{g, n-1}, x\right) \rightarrow \mathrm{H}_{1}^{P^{\prime}}\left(\Sigma_{g, n-1}\right)$.

Remark The splitting of $\pi_{1}\left(U \Sigma_{g, n-1}\right)$ from the second part of Theorem 4.1 is not natural. If $\overline{\mathrm{H}}_{1}^{P^{\prime}}\left(\Sigma_{g, n-1}\right)=\mathrm{H}_{1}\left(\Sigma_{g, n-1}\right)$ (so $K^{\prime}$ is the commutator subgroup), then since exact sequence (2) describes a central extension, the embedding of $K^{\prime}$ into $\pi_{1}\left(U \Sigma_{g, n-1}\right)$ is canonical. However, this need not be the case. 
The proof of the first part of this theorem is easy.

Proof of Theorem 4.1 when $\boldsymbol{p}=\{\boldsymbol{b}\} \quad$ Let $i: \Sigma_{g, n} \hookrightarrow \Sigma_{g, n-1}$ be the embedding. Observe that since $p=\{b\}$, the map $i$ induces an isomorphism between $\mathrm{H}_{1}^{P}\left(\Sigma_{g, n}\right)$ and $\mathrm{H}_{1}^{P^{\prime}}\left(\Sigma_{g, n-1}\right)$. This isomorphism is equivariant with respect to the action of the two mapping class groups, so Theorem 3.3 implies that $f \in \mathcal{I}\left(\Sigma_{g, n}, P\right)$ if and only if $i_{*}(f) \in \mathcal{I}\left(\Sigma_{g, n-1}, P^{\prime}\right)$. This implies that the standard Birman exact sequence restricts in the indicated manner, as desired.

The proof of the other part of Theorem 4.1 is somewhat more complicated. Our proof makes use of some ideas of van den Berg [20, Proposition 2.4.1].

Proof of Theorem 4.1 when $\boldsymbol{p} \neq\{\boldsymbol{b}\}$ Observe that since $p \neq\{b\}$, we must have $n-1 \geq 1$. Thus $\pi_{1}\left(\Sigma_{g, n-1}, x\right)$ is a free group and exact sequence (2) splits, so $\pi_{1}\left(U \Sigma_{g, n-1}, \tilde{x}\right) \cong \pi_{1}\left(\Sigma_{g, n-1}, x\right) \oplus \mathbb{Z}$. Fix such a splitting. We begin with a criterion for an element of $\operatorname{Mod}\left(\Sigma_{g, n}\right)$ to lie in $\mathcal{I}\left(\Sigma_{g, n}, P\right)$. Let $h \in \mathrm{H}_{1}^{P}\left(\Sigma_{g, n}\right)$ be the homology class of any arc between $b$ and some other boundary component.

Claim 1 Consider $f \in \operatorname{Mod}\left(\Sigma_{g, n}\right)$. Assume that $i_{*}(f) \in \mathcal{I}\left(\Sigma_{g, n-1}, P^{\prime}\right)$ and that $f$ fixes the homology class $h$. Then $f \in \mathcal{I}\left(\Sigma_{g, n}, P\right)$.

Proof of claim 1 Define $L \subset \mathrm{H}_{1}^{P}\left(\Sigma_{g, n}\right)$ to equal

$$
\begin{aligned}
\left\langle\left\{[g] \in \mathrm{H}_{1}^{P}\left(\Sigma_{g, n}\right) \mid\right.\right. & g \text { is a simple closed curve or a properly embedded } \\
& \text { arc between } \left.\left.P^{\prime} \text {-adjacent boundary components }\right\}\right\rangle .
\end{aligned}
$$

It is easy to see that $\mathrm{H}_{1}^{P}\left(\Sigma_{g, n}\right)=\langle L, h\rangle$, so it is enough to prove that $f$ fixes $L$. Observe that

$$
\mathrm{H}_{1}^{P^{\prime}}\left(\Sigma_{g, n-1}\right) \cong L /\langle b\rangle \text {. }
$$

Since $i_{*}(f) \in \mathcal{I}\left(\Sigma_{g, n-1}, P^{\prime}\right)$, it follows that for $g \in L$ we have $f_{*}(g)=g+k \cdot[b]$ for some integer $k$ (the integer $k$ depends on $g$ ). However,

$$
i_{a}(h, g)=i_{a}\left(f_{*}(h), f_{*}(g)\right)=i_{a}(h, g+k \cdot[b])=i_{a}(h, g)+k .
$$

This implies that $k=0$, as desired.

We now examine the manner in which $\pi_{1}\left(U \Sigma_{g, n-1}, \tilde{x}\right) \subset \operatorname{Mod}\left(\Sigma_{g, n}\right)$ acts on $h$. Observe that we have an injection

$$
j: \mathrm{H}_{1}^{P^{\prime}}\left(\Sigma_{g, n-1}\right) \hookrightarrow \mathrm{H}_{1}^{P}\left(\Sigma_{g, n}\right) /\langle[b]\rangle .
$$


Since $\operatorname{Mod}\left(\Sigma_{g, n}\right)$ preserves $[b]$, the action of $\operatorname{Mod}\left(\Sigma_{g, n}\right)$ on $\mathrm{H}_{1}^{P}\left(\Sigma_{g, n}\right)$ descends to an action on $\mathrm{H}_{1}^{P}\left(\Sigma_{g, n}\right) /\langle[b]\rangle$. Recall that $T_{b}$ is the generator of the kernel of exact sequence (2). Since $T_{b}$ acts trivially on $\mathrm{H}_{1}^{P}\left(\Sigma_{g, n}\right) /\langle[b]\rangle$, exact sequence (2) implies that the action of $\pi_{1}\left(U \Sigma_{g, n-1}, \tilde{x}\right)$ on $\mathrm{H}_{1}^{P}\left(\Sigma_{g, n}\right) /\langle[b]\rangle$ descends to an action of $\pi_{1}\left(\Sigma_{g, n-1}, x\right)$ on $\mathrm{H}_{1}^{P}\left(\Sigma_{g, n}\right) /\langle[b]\rangle$. Let $\bar{h}$ be the image of $h$ in $\mathrm{H}_{1}^{P}\left(\Sigma_{g, n}\right) /\langle[b]\rangle$. The following claim says that $\pi_{1}\left(\Sigma_{g, n-1}, x\right)$ acts in the most obvious possible way on $\bar{h}$ :

Claim $2 \gamma \in \pi_{1}\left(\Sigma_{g, n-1}, x\right)$ acts upon $\bar{h}$ by

$$
\bar{h} \longmapsto \bar{h}+j([\gamma]) \text {. }
$$

Proof of claim 2 Assume first that $\gamma$ is a simple closed curve. In this case, the lift $\tilde{\sigma}_{\gamma} \in \operatorname{Mod}\left(\Sigma_{g, n}\right)$ (see Figure 2.b) equals $T_{\tilde{\gamma}_{1}} T_{\tilde{\gamma}_{2}}^{-1}$ for two simple closed curve $\tilde{\gamma}_{1}$ and $\tilde{\gamma}_{2}$ satisfying

$$
\left[\tilde{\gamma}_{2}\right]=\left[\tilde{\gamma}_{1}\right]+[b] .
$$

Now, we know that $i_{a}(h,[b])=1$. The claim then follows from an easy calculation, using the fact that Dehn twists act as transvections on homology.

To prove the general case, observe that by the proof of the case $p=\{b\}$, we know that $\pi_{1}\left(U \Sigma_{g, n}, \tilde{x}\right)$ acts as the identity on the submodule of $\mathrm{H}_{1}^{P}\left(\Sigma_{g, n}\right)$ generated by the homology classes of simple closed curves. Now, since simple closed curves generate $\pi_{1}\left(\Sigma_{g, n-1}, x\right)$, we can write $\gamma=\gamma_{1} \gamma_{2} \cdots \gamma_{k}$, where the $\gamma_{i}$ are simple closed curves. The previous case (plus the observation at the beginning of this paragraph) then implies that

$$
\begin{aligned}
\gamma(\bar{h}) & =\left(\gamma_{1} \gamma_{2} \cdots \gamma_{k}\right)(\bar{h})=\left(\gamma_{1} \gamma_{2} \cdots \gamma_{k-1}\right)\left(\bar{h}+j\left(\left[\gamma_{k}\right]\right)\right) \\
& =\left(\gamma_{1} \gamma_{2} \cdots \gamma_{k-2}\right)\left(\bar{h}+j\left(\left[\gamma_{k-1}\right]\right)+j\left(\left[\gamma_{k}\right]\right)\right) \\
& =\cdots=\bar{h}+j\left(\left[\gamma_{1}\right]\right)+\cdots+j\left(\left[\gamma_{k}\right]\right)=\bar{h}+j([\gamma]),
\end{aligned}
$$

as desired.

We now prove that $K$ equals the graph of some subgroup $K^{\prime} \subset \pi_{1}\left(\Sigma_{g, n-1}, x\right)$.

Claim 3 There exists a subgroup $K^{\prime}$ of $\pi_{1}\left(\Sigma_{g, n-1}, x\right)$ and a homomorphism $\phi: K^{\prime} \rightarrow$ $\mathbb{Z}$ so that $K$ is the graph of $\phi$. 
Proof of claim 3 Let $K^{\prime}$ equal the projection of $K \subset \pi_{1}\left(\Sigma_{g, n-1}, x\right) \oplus \mathbb{Z}$ to $\pi_{1}\left(\Sigma_{g, n-1}, x\right)$. To prove that $K$ is the graph of a homomorphism $\phi: K^{\prime} \rightarrow \mathbb{Z}$, it is enough to show that each element of $K^{\prime}$ is the image of exactly one element of $K$. In other words, we must prove that $\mathbb{Z} \cap K=1$. This follows from the fact that $T_{b}^{m}(h)=h+m[b] \neq h$ if $m \neq 0$.

We now identify $K^{\prime}$.

Claim $4 K^{\prime}$ equals the kernel of the natural map $\pi_{1}\left(\Sigma_{g, n-1}, x\right) \rightarrow \mathrm{H}_{1}^{P^{\prime}}\left(\Sigma_{g, n-1}\right)$.

Proof of claim 4 Let $K^{\prime \prime}$ be the kernel of the natural map $\pi_{1}\left(\Sigma_{g, n-1}, x\right) \rightarrow$ $\mathrm{H}_{1}^{P^{\prime}}\left(\Sigma_{g, n-1}\right)$, and consider $f \in \pi_{1}\left(U \Sigma_{g, n-1}, \tilde{x}\right)$. Let $\bar{f} \in \pi_{1}\left(\Sigma_{g, n-1}, x\right)$ be the projection of $f$. To show that $K^{\prime}=K^{\prime \prime}$, we need to show that there exists some $m \in \mathbb{Z}$ so that $f T_{b}^{m} \in \mathcal{I}\left(\Sigma_{g, n}, P\right)$ if and only if $\bar{f} \in K^{\prime \prime}$. By Claim $1, f T_{b}^{m} \in \mathcal{I}\left(\Sigma_{g, n}, P\right)$ if and only if $f T_{b}^{m}$ fixes the homology class $h$. Now, $T_{b}^{m}(h)=h+m[b]$. It follows that we can find an $m$ such that $f T_{b}^{m}$ fixes the homology class $h$ if and only if $f$ fixes $\bar{h} \in \mathrm{H}_{1}^{P}\left(\Sigma_{g, n}\right) /\langle[b]\rangle$ (see the discussion before Claim 2). By Claim 2, this will be true if and only if $\bar{f} \in K^{\prime \prime}$, as desired.

We finish by proving that the map $\mathcal{I}\left(\Sigma_{g, n}, P\right) \rightarrow \mathcal{I}\left(\Sigma_{g, n-1}, P^{\prime}\right)$ is surjective.

Claim 5 Let $\bar{f} \in \mathcal{I}\left(\Sigma_{g, n-1}, P^{\prime}\right)$. There then exists some $f \in \mathcal{I}\left(\Sigma_{g, n}, P\right)$ so that $i_{*}(f)=\bar{f}$.

Proof of claim 5 Let $f \in \operatorname{Mod}\left(\Sigma_{g, n}\right)$ be any lift of $\bar{f}$. Since the space of all embeddings of $h \cup b$ into $\Sigma_{g, n-1}$ which fix the endpoint of $h$ not on $b$ is connected, we can assume that $f$ fixes $h$. Claim 5 then tells us that $f \in \mathcal{I}\left(\Sigma_{g, n}, P\right)$, as desired. $\square$

This completes the proof of the theorem.

\subsection{An addendum to Theorem 4.1}

We now prove the following lemma, whose proof uses some ideas from the proof of [20, Proposition 2.4.2]:

Lemma 4.2 Let

$$
1 \longrightarrow K \longrightarrow \mathcal{I}\left(\Sigma_{g, n}, P\right) \longrightarrow \mathcal{I}\left(\Sigma_{g, n-1}, P^{\prime}\right) \longrightarrow 1
$$




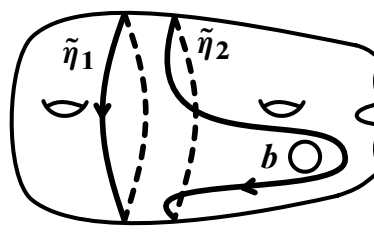

a

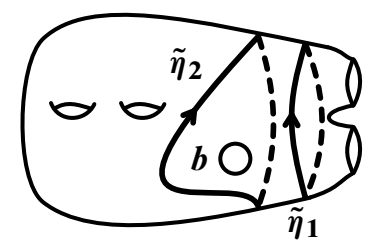

b

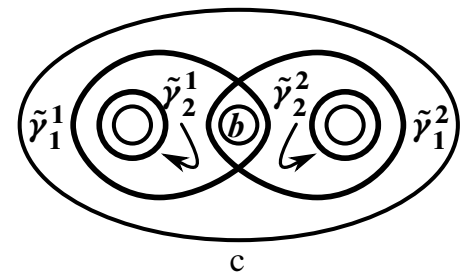

Figure 5: a: $\tilde{\sigma}_{\eta}$ for a simple closed curve $\eta$ which cuts off a one-holed torus

b: $\tilde{\sigma}_{\eta}$ for a simple closed curve $\eta$ which cuts off a set of boundary components

c: $\tilde{\sigma}_{\gamma^{1}}$ and $\tilde{\sigma}_{\gamma^{2}}$ for simple closed curves $\gamma^{1}$ and $\gamma^{2}$ so that $\gamma^{1} \cap \gamma^{2}=\{x\}$ and so that a regular neighborhood of $\gamma^{1} \cup \gamma^{2}$ is homeomorphic to $\Sigma_{0,3}$.

be as in Theorem 4.1. If $g \geq 1$, then $K$ is in the subgroup of $\mathcal{I}\left(\Sigma_{g, n}, P\right)$ generated by twists about $P$-separating curves and twists about $P$-bounding pairs. If $g=0$, then $K$ is in the subgroup generated by twists about $P$-separating curves, twists about $P$-bounding pairs, and commutators of simply intersecting pairs (see the introduction for the definition of a commutator of a simply intersecting pair).

Proof As in Theorem 4.1, let $b$ be the boundary component to which we are attaching a disc and $p \in P$ be the partition element containing $b$. If $p=\{b\}$, then the lemma is trivial, so we assume that $p \neq\{b\}$. This implies that $K$ is isomorphic to the kernel of the natural map $\pi_{1}\left(\Sigma_{g, n-1}\right) \rightarrow \mathrm{H}_{1}^{P^{\prime}}\left(\Sigma_{g, n-1}\right)$. This kernel is generated by the commutator subgroup $\left[\pi_{1}\left(\Sigma_{g, n-1}\right), \pi_{1}\left(\Sigma_{g, n-1}\right)\right]$ plus the set of all simple closed curves which cut off subsets $q \in P^{\prime}$ of boundary components. We will prove that these generators lie in the indicated subgroup of $\mathcal{I}\left(\Sigma_{g, n}, P\right)$.

We begin with the commutator subgroup. If $g \geq 1$, then Lemma A.1 from the appendix says that the commutator subgroup is generated by $\left[\gamma^{1}, \gamma^{2}\right]$, where $\gamma^{1}, \gamma^{2} \in$ $\pi_{1}\left(\Sigma_{g, n-1}\right)$ are simple closed curves which only intersect at the basepoint and where a regular neighborhood of $\gamma^{1} \cup \gamma^{2}$ is homeomorphic to $\Sigma_{1,1}$. This implies that $\left[\gamma^{1}, \gamma^{2}\right]$ is homotopic to a simple closed curve $\eta$ which cuts off a one-holed torus. Replacing $\eta$ by its inverse if necessary, the lift $\tilde{\sigma}_{\eta}$ then equals $T_{\tilde{\eta}_{1}} T_{\tilde{\eta}_{2}}^{-1}$ for simple closed curves $\tilde{\eta}_{1}$ and $\tilde{\eta}_{2}$ like those depicted in Figure 5.a. Observe that $T_{\tilde{\eta}_{1}}$ is a twist about a $P$-separating curve and that $T_{b} T_{\tilde{\eta}_{2}}^{-1}$ is a twist about a $P$-bounding pair. We conclude that the element of $\mathcal{I}\left(\Sigma_{g, n}, P\right)$ associated to $\left[\gamma^{1}, \gamma^{2}\right]$ equals

$$
\tilde{\sigma}_{\eta} T_{b}=T_{\tilde{\eta}_{1}}\left(T_{b} T_{\tilde{\eta}_{2}}^{-1}\right),
$$

which is in the desired subgroup. 
If $g=0$, then pairs of curves like those in the previous paragraph do not exist. However, it is immediate that the commutator subgroup is generated by $\left[\gamma^{1}, \gamma^{2}\right]$, where $\gamma^{1}, \gamma^{2} \in \pi_{1}\left(\Sigma_{g, n-1}\right)$ are simple closed curves which only intersect at the basepoint and where a regular neighborhood of $\gamma^{1} \cup \gamma^{2}$ is homeomorphic to $\Sigma_{0,3}$. In this case, for $1 \leq i \leq 2$ we have that $\tilde{\sigma}_{\gamma^{i}}=T_{\tilde{\gamma}_{1}^{i}}^{ \pm 1} T_{\tilde{\gamma}_{2}^{i}}^{\mp 1}$ for curves like those depicted in Figure 5.c (the signs depend on the orientations on $\gamma^{1}$ and $\gamma^{2}$ ). Observe that

$$
\left[T_{\tilde{\gamma}_{1}^{1}}^{ \pm 1} T_{\tilde{\gamma}_{2}^{1}}^{\mp 1}, T_{\tilde{\gamma}_{1}^{2}}^{ \pm 1} T_{\tilde{\gamma}_{2}^{2}}^{\mp 1}\right]=\left[T_{\tilde{\gamma}_{1}^{1}}^{ \pm 1}, T_{\tilde{\gamma}_{1}^{2}}^{ \pm 1}\right] .
$$

Using the commutator identities $\left[g_{1}^{-1}, g_{2}\right]=\left[g_{2}, g_{1}\right]^{g_{1}^{-1}}$ and $\left[g_{1}, g_{2}^{-1}\right]=\left[g_{2}, g_{1}\right]_{2}^{g_{2}^{-1}}$ if necessary, we see that this is a commutator of a simply intersecting pair, thus proving that the element of $\mathcal{I}\left(\Sigma_{g, n}, P\right)$ associated to $\left[\gamma^{1}, \gamma^{2}\right]$ lies in the desired subgroup.

We conclude by considering a simple closed curve $\eta \in \pi_{1}\left(\Sigma_{g, n-1}, x\right)$ which cuts off a subset $q \in P^{\prime}$ of boundary components. Reversing the orientation of $\eta$ if necessary, the associated element $\tilde{\sigma}_{\eta}$ of $\operatorname{Mod}\left(\Sigma_{g, n}\right)$ equals $T_{\tilde{\eta}_{1}} T_{\tilde{\eta}_{2}}^{-1}$ for the curves $\tilde{\eta}_{1}$ and $\tilde{\eta}_{2}$ pictured in Figure 5.b. There are two cases. The first case is $q \neq p \backslash\{b\}$. In this case, $T_{\tilde{\eta}_{1}}$ is a $P$-separating curve and $T_{b} T_{\tilde{\eta}_{2}}^{-1}$ is a $P$-bounding pair. Hence the element of $\mathcal{I}\left(\Sigma_{g, n}, P\right)$ associated to $\eta$ equals

$$
\tilde{\sigma}_{\eta} T_{b}=T_{\tilde{\eta}_{1}}\left(T_{b} T_{\tilde{\eta}_{2}}^{-1}\right),
$$

which is in the desired subgroup. The other case is $q=p \backslash\{b\}$. In this case, $T_{\tilde{\eta}_{2}}$ is a $P$-separating curve and $T_{\tilde{\eta}_{1}} T_{b}^{-1}$ is a $P$-bounding pair. Hence the element of $\mathcal{I}\left(\Sigma_{g, n}, P\right)$ associated to $\eta$ is

$$
\tilde{\sigma}_{\eta} T_{b}^{-1}=\left(T_{\tilde{\eta}_{1}} T_{b}^{-1}\right) T_{\tilde{\eta}_{2}}^{-1},
$$

which again is in the desired subgroup.

\section{Generators for the Torelli groups}

In this section, we will prove Theorems 1.3 and 1.5. Our main tool will be Theorem 2.1. We will apply this theorem to the action of the Torelli group on the complex of curves, which is a simplicial complex introduced by Harvey [10] to encode the combinatorial topology of a surface.

Definition The complex of curves $\mathcal{C}\left(\Sigma_{g}\right)$ is the simplicial complex whose simplices are sets $\left\{\gamma_{1}, \ldots, \gamma_{k}\right\}$ of isotopy classes of nontrivial (that is, not isotopic to a point) simple closed curves which can be realized disjointly. 
The following theorem is due to Harer [9]; see [11] for an alternate proof:

Theorem $5.1 \mathcal{C}\left(\Sigma_{g}\right)$ is $2 g-3$-connected. In particular, $\mathcal{C}\left(\Sigma_{g}\right)$ is simply-connected for $g \geq 2$.

Observe that $\mathcal{I}\left(\Sigma_{g}\right)$ acts on $\mathcal{C}\left(\Sigma_{g}\right)$. A theorem of Ivanov [12, Theorem 1.2] implies the following:

Theorem 5.2 For all $g$, the group $\mathcal{I}\left(\Sigma_{g}\right)$ acts on $\mathcal{C}\left(\Sigma_{g}\right)$ without rotations.

The following theorem will be the key to our argument:

Theorem 5.3 For $g \geq 2$, the complex $\mathcal{C}\left(\Sigma_{g}\right) / \mathcal{I}\left(\Sigma_{g}\right)$ is simply-connected.

We postpone the proof of Theorem 5.3 until Section 6. Instead, we use it to prove Theorems 1.3 and 1.5 .

Proof of Theorems 1.3 and 1.5 Observe first that Theorem 1.5 follows from repeated applications of Lemma 4.2. We will prove Theorem 1.3 by induction on $g$. The base case is $g=1$. Using Lemma 4.2, we can assume that $n=0$, in which case the theorem is trivial.

Now consider a partitioned surface $\left(\Sigma_{g, n}, P\right)$ with $g>1$. By repeated use of Lemma 4.2, we can reduce to the case that $n=0$ (and hence forget about $P$ ). By Theorems 5.1, 5.3, and 5.2, we can use Theorem 2.1 to conclude that $\mathcal{I}\left(\Sigma_{g}\right)$ is generated by the subgroups $\Gamma_{\gamma}$ of $\mathcal{I}\left(\Sigma_{g}\right)$ stabilizing simple closed curves $\gamma$. If $\gamma$ is a separating curve which separates $\Sigma_{g}$ into two surfaces $\Sigma_{h_{1}, 1}$ and $\Sigma_{h_{2}, 1}$ with $h_{1}+h_{2}=g$, then we have an exact sequence

$$
1 \longrightarrow\left\langle T_{\gamma}\right\rangle \longrightarrow \mathcal{I}\left(\Sigma_{h_{1}, 1}\right) \oplus \mathcal{I}\left(\Sigma_{h_{2}, 1}\right) \longrightarrow \Gamma_{\gamma} \longrightarrow 1 .
$$

By induction, both $\Sigma_{h_{1}, 1}$ and $\Sigma_{h_{2}, 1}$ are generated by twists about separating curves and bounding pairs, so we conclude that $\Gamma_{\gamma}$ is also generated by such elements, as desired. If instead $\gamma$ is a nonseparating curve, let $N$ be a small open regular neighborhood of $\gamma$. The surface $S=\Sigma_{g} \backslash N$ is then a genus $g-1$ surface with 2 boundary components $b_{1}$ and $b_{2}$, and additionally the inclusion $S \hookrightarrow \Sigma_{g}$ is a capping of $(S, P)$ for the partition $P=\left\{\left\{b_{1}, b_{2}\right\}\right\}$ of the boundary components of $S$. By induction, the group $\mathcal{I}(S, P)$ is generated by $P$-separating curves and $P$-bounding pairs. Now, we have an exact sequence

$$
1 \longrightarrow\left\langle T_{b_{1}} T_{b_{2}}^{-1}\right\rangle \longrightarrow \mathcal{I}(S, P) \longrightarrow \Gamma_{\gamma} \longrightarrow 1 .
$$

Since the $P$-separating curves and $P$-bounding pairs in $\mathcal{I}(S, P)$ project to separating curves and bounding pairs in $\Gamma_{\gamma}$, we conclude that $\Gamma_{\gamma}$ is generated by such elements, and we are done. 


\section{The connectivity of $\mathcal{C}\left(\Sigma_{g}\right) / \mathcal{I}\left(\Sigma_{g}\right)$}

In this section, we prove Theorem 5.3. First, in Section 6.1 we interpret a large subcomplex of $\mathcal{C}\left(\Sigma_{g}\right) / \mathcal{I}\left(\Sigma_{g}\right)$ in terms of $\mathrm{H}_{1}\left(\Sigma_{g}\right)$. Next, in Section 6.2 we show how to homotope loops in $\mathcal{C}\left(\Sigma_{g}\right) / \mathcal{I}\left(\Sigma_{g}\right)$ so that they "avoid" a fixed homology class. In Section 6.3 this leads quickly to a proof of Theorem 5.3 when $g \geq 3$. In the genus 2 case, the additional argument needed is given in Section 6.4.

\subsection{A concrete description of a subcomplex of $\mathcal{C}\left(\Sigma_{g}\right) / \mathcal{I}\left(\Sigma_{g}\right)$}

In this section, we give a concrete description of the quotient by $\mathcal{I}\left(\Sigma_{g}\right)$ of the following subcomplex of $\mathcal{C}\left(\Sigma_{g}\right)$ :

Definition The nonseparating complex of curves on $\Sigma_{g}$, which we will denote $\mathcal{C}_{\text {nosep }}\left(\Sigma_{g}\right)$, is the subcomplex of $\mathcal{C}\left(\Sigma_{g}\right)$ whose simplices are sets $\left\{\gamma_{1}, \ldots, \gamma_{k}\right\}$ of isotopy classes of simple closed curves on $\Sigma_{g}$ so that $\Sigma_{g} \backslash\left(\gamma_{1} \cup \cdots \cup \gamma_{k}\right)$ is connected.

The importance of this subcomplex for us comes from the following:

Lemma 6.1 The inclusion $\mathcal{C}_{\text {nosep }}\left(\Sigma_{g}\right) / \mathcal{I}\left(\Sigma_{g}\right) \hookrightarrow \mathcal{C}\left(\Sigma_{g}\right) / \mathcal{I}\left(\Sigma_{g}\right)$ induces a surjection on $\pi_{1}$.

Proof Fix a base point in $\mathcal{C}\left(\Sigma_{g}\right) / \mathcal{I}\left(\Sigma_{g}\right)$ at a vertex corresponding to a nonseparating curve, and consider a based loop $\ell \in \pi_{1}\left(\mathcal{C}\left(\Sigma_{g}\right) / \mathcal{I}\left(\Sigma_{g}\right)\right)$. We can assume that $\ell$ is a simplicial path in the 1 -skeleton. Lift $\ell$ (one edge at a time) to a path $\tilde{\ell}$ in $\mathcal{C}\left(\Sigma_{g}\right)$. Assume that $\tilde{\ell}$ contains a subpath of the form $\gamma_{1}-\gamma_{2}-\gamma_{3}$, where $\gamma_{2}$ is a separating curve. If $\gamma_{1}$ and $\gamma_{3}$ lie on different sides of $\gamma_{2}$, then they are disjoint and we can replace $\gamma_{1}-\gamma_{2}-\gamma_{3}$ with $\gamma_{1}-\gamma_{3}$. Otherwise, let $\gamma_{2}^{\prime}$ be a nonseparating curve on the side of $\gamma_{2}$ not containing $\gamma_{1}$ and $\gamma_{3}$. We can then homotope our segment to $\gamma_{1}-\gamma_{2}^{\prime}-\gamma_{3}$, eliminating the separating curve $\gamma_{2}$. A similar argument allows us to eliminate any edges corresponding to pairs of nonseparating curves which together separate the surface. This allows us to homotope $\tilde{\ell}$ into the 1 -skeleton of $\mathcal{C}_{\text {nosep }}\left(\Sigma_{g}\right)$. Projecting this homotopy to $\mathcal{C}\left(\Sigma_{g}\right) / \mathcal{I}\left(\Sigma_{g}\right)$, the based loop $\ell$ is homotoped to a loop coming from a loop in $\mathcal{C}_{\text {nosep }}\left(\Sigma_{g}\right) / \mathcal{I}\left(\Sigma_{g}\right)$, as desired.

Now, consider a simplex $\left\{\alpha_{1}, \ldots, \alpha_{k}\right\}$ of $\mathcal{C}_{\text {nosep }}\left(\Sigma_{g}\right)$. Observe that since $\Sigma_{g} \backslash\left(\alpha_{1} \cup\right.$ $\left.\cdots \cup \alpha_{k}\right)$ is connected, we can find nonseparating curves $\left\{\alpha_{k+1}, \ldots, \alpha_{g}, \beta_{1}, \ldots, \beta_{g}\right\}$ so that $i_{g}\left(\alpha_{i}, \alpha_{j}\right)=i_{g}\left(\beta_{i}, \beta_{j}\right)=0$ and $i_{g}\left(\alpha_{i}, \beta_{j}\right)=\delta_{i j}$ for all $i$ and $j$ and so that $\Sigma_{g} \backslash\left(\alpha_{1} \cup \beta_{1} \cup \cdots \cup \alpha_{g} \cup \beta_{g}\right)$ is connected. If we orient the $\alpha_{i}$ and the $\beta_{j}$ correctly, this 
implies that $\left\{\left[\alpha_{1}\right],\left[\beta_{1}\right], \ldots,\left[\alpha_{g}\right],\left[\beta_{g}\right]\right\}$ is a symplectic basis for $\mathrm{H}_{1}\left(\Sigma_{g}\right)$. In particular, $\left\{\left[\alpha_{1}\right], \ldots,\left[\alpha_{k}\right]\right\}$ spans a $k$-dimensional isotropic summand of $\mathrm{H}_{1}\left(\Sigma_{g}\right)$ (a summand $A$ of $\mathrm{H}_{1}\left(\Sigma_{g}\right)$ is isotropic if $i_{a}(x, y)=0$ for all $\left.x, y \in A\right)$. Since we had to put arbitrary orientations on the $\alpha_{i}$ and the $\beta_{j}$, this suggests the following definition:

Definition Let $V$ be a $\mathbb{Z}$-module with a symplectic form $i(\cdot, \cdot)$. The complex of unimodular isotropic lines in $V$, denoted $\mathcal{L}(V)$, is the simplicial complex whose simplices are sets $\left\{L_{1}, \ldots, L_{k}\right\}$ of 1 -dimensional summands $L_{i}$ of $V$ so that $\left\langle L_{1}, \ldots, L_{k}\right\rangle$ is a $k$-dimensional isotropic summand of $V$.

Since the Torelli group preserves $\mathrm{H}_{1}\left(\Sigma_{g}\right)$, there is a natural map

$$
\pi: \mathcal{C}_{\text {nosep }}\left(\Sigma_{g}\right) / \mathcal{I}\left(\Sigma_{g}\right) \longrightarrow \mathcal{L}\left(\mathrm{H}_{1}\left(\Sigma_{g}\right)\right) .
$$

We will prove the following:

Lemma 6.2 $\pi$ is an isomorphism of simplicial complexes.

Proof We have a series of projections

$$
\mathcal{C}_{\text {nosep }}\left(\Sigma_{g}\right) \stackrel{\tilde{\pi}}{\longrightarrow} \mathcal{C}_{\text {nosep }}\left(\Sigma_{g}\right) / \mathcal{I}\left(\Sigma_{g}\right) \stackrel{\pi}{\longrightarrow} \mathcal{L}\left(\mathrm{H}_{1}\left(\Sigma_{g}\right)\right) .
$$

We must prove that for all simplices $s$ of $\mathcal{L}\left(\mathrm{H}_{1}\left(\Sigma_{g}\right)\right)$, there is some simplex $\tilde{s}$ of $\mathcal{C}_{\text {nosep }}\left(\Sigma_{g}\right)$ so that $\pi \circ \tilde{\pi}(\tilde{s})=s$, and in addition if $\tilde{s}_{1}$ and $\tilde{s}_{2}$ are simplices of $\mathcal{C}_{\text {nosep }}\left(\Sigma_{g}\right)$ so that $\pi \circ \tilde{\pi}\left(\tilde{s}_{1}\right)=\pi \circ \tilde{\pi}\left(\tilde{s}_{2}\right)$, then there is some $f \in \mathcal{I}\left(\Sigma_{g}\right)$ so that $f\left(\tilde{s}_{1}\right)=\tilde{s}_{2}$. We begin with the first assertion. Let $s$ be a simplex of $\mathcal{L}\left(\mathrm{H}_{1}\left(\Sigma_{g}\right)\right)$. Pick a symplectic basis $\left\{a_{1}, b_{1}, \ldots, a_{g}, b_{g}\right\}$ so that $s=\left\{\left\langle a_{1}\right\rangle, \ldots,\left\langle a_{h}\right\rangle\right\}$. Lemma A.3 from the appendix allows us to realize this symplectic basis by simple closed curves $\left\{\alpha_{1}, \beta_{1}, \ldots, \alpha_{g}, \beta_{g}\right\}$. Observe that $\tilde{s}=\left\{\alpha_{1}, \ldots, \alpha_{h}\right\}$ is a simplex of $\mathcal{C}_{\text {nosep }}\left(\Sigma_{g}\right)$ with $\pi \circ \tilde{\pi}(\tilde{s})=s$.

We now prove the section assertion. Let $\tilde{s}_{1}$ and $\tilde{s}_{2}$ be two simplices of $\mathcal{C}_{\text {nosep }}\left(\Sigma_{g}\right)$ with $\pi \circ \tilde{\pi}\left(\tilde{s}_{1}\right)=\pi \circ \tilde{\pi}\left(\tilde{s}_{2}\right)$. Let the vertices of the $\tilde{s}_{i}$ be $\left\{\alpha_{1}^{i}, \ldots, \alpha_{h}^{i}\right\}$. Order these and pick orientations so that $\left[\alpha_{j}^{1}\right]=\left[\alpha_{j}^{2}\right]$. Set $a_{j}=\left[\alpha_{j}^{1}\right]$, and extend this to a symplectic basis $\left\{a_{1}, b_{1} \ldots, a_{g}, b_{g}\right\}$ for homology. Lemma A.3 from the appendix allows us to extend $\left\{\alpha_{1}^{i}, \ldots, \alpha_{h}^{i}\right\}$ to a set of oriented simple closed curves $\left\{\alpha_{1}^{i}, \beta_{1}^{i}, \ldots, \alpha_{g}^{i}, \beta_{g}^{i}\right\}$ realizing the homology basis $\left\{a_{1}, b_{1} \ldots, a_{g}, b_{g}\right\}$. Using the classification of surfaces, there must exist some $f \in \operatorname{Mod}\left(\Sigma_{g}\right)$ so that $f\left(\alpha_{j}^{1}\right)=\alpha_{j}^{2}$ and $f\left(\beta_{j}^{1}\right)=\beta_{j}^{2}$ for all $j$. Since we have chosen $f$ so that it fixes a basis for homology, it follows that $f \in \mathcal{I}\left(\Sigma_{g}\right)$. The proof concludes with the observation that $f\left(\tilde{s}_{1}\right)=\tilde{s}_{2}$.

Henceforth we will identify $\mathcal{C}_{\text {nosep }}\left(\Sigma_{g}\right) / \mathcal{I}\left(\Sigma_{g}\right)$ with $\mathcal{L}\left(\mathrm{H}_{1}\left(\Sigma_{g}\right)\right)$. 


\subsection{Homotoping loops off a homology class}

In this section, we will show how to homotope (in $\mathcal{C}\left(\Sigma_{g}\right) / \mathcal{I}\left(\Sigma_{g}\right)$ ) curves which lie in $\mathcal{L}\left(\mathrm{H}_{1}\left(\Sigma_{g}\right)\right)$ so that they "avoid" a fixed homology class. To make this precise, we need the following definition:

Definition Let $V$ be a $\mathbb{Z}$-module with a symplectic form $i(\cdot, \cdot)$, and let $W$ be a submodule of $V$. We then define $\mathcal{L}^{W}(V)$ to be the full subcomplex of $\mathcal{L}(V)$ whose vertices are 1-dimensional summands $L$ of $V$ so that $L \subset W$.

We will also need the following standard definition from PL-topology:

Definition Let $v$ be a vertex of a simplicial complex $X$. The link of $v$ in $X$ (denoted $\left.\operatorname{link}_{v}(X)\right)$ is the subcomplex of $X$ whose simplices are simplices $s$ of $X$ so that $v \notin s$ and so that $s \cup\{v\}$ is a simplex of $X$.

Fix a symplectic basis $\left\{a_{1}, b_{1}, \ldots, a_{g}, b_{g}\right\}$ for $\mathrm{H}_{1}\left(\Sigma_{g}\right)$, and set

$$
W=\left\langle a_{1}, b_{1}, \ldots, a_{g-1}, b_{g-1}, a_{g}\right\rangle \subset \mathrm{H}_{1}\left(\Sigma_{g}\right) .
$$

Our lemma is the following:

Lemma 6.3 Let $i: \mathcal{L}\left(\mathrm{H}_{1}\left(\Sigma_{g}\right)\right) \hookrightarrow \mathcal{C}\left(\Sigma_{g}\right) / \mathcal{I}\left(\Sigma_{g}\right)$ be the inclusion.

- For $g \geq 2$, let $\ell$ be a simplicial arc in $\mathcal{L}\left(\mathrm{H}_{1}\left(\Sigma_{g}\right)\right)$ whose endpoints lie in $\mathcal{L}^{W}\left(\mathrm{H}_{1}\left(\Sigma_{g}\right)\right)$. There is then a simplicial arc $\ell^{\prime}$ in $\mathcal{L}^{W}\left(\mathrm{H}_{1}\left(\Sigma_{g}\right)\right)$ so that $i_{*}(\ell)$ is homotopic to $i_{*}\left(\ell^{\prime}\right)$ (fixing the endpoints).

- For $g \geq 3$ and $L$ any vertex of $\mathcal{L}\left(\mathrm{H}_{1}\left(\Sigma_{g}\right)\right)$, let $\ell$ be a simplicial arc in $\operatorname{link}_{L}\left(\mathcal{L}\left(\mathrm{H}_{1}\left(\Sigma_{g}\right)\right)\right)$ whose endpoints lie in $\mathcal{L}^{W}\left(\mathrm{H}_{1}\left(\Sigma_{g}\right)\right)$. There is then a simplicial arc $\ell^{\prime}$ in $\mathcal{L}^{W}\left(\mathrm{H}_{1}\left(\Sigma_{g}\right)\right) \cap \operatorname{link}_{L}\left(\mathcal{L}\left(\mathrm{H}_{1}\left(\Sigma_{g}\right)\right)\right)$ so that $i_{*}(\ell)$ is homotopic to $i_{*}\left(\ell^{\prime}\right)$ (fixing the endpoints).

An immediate corollary of the first conclusion of this lemma and Lemma 6.1 is the following:

Corollary 6.4 For $g \geq 2$, the inclusion $\mathcal{L}^{W}\left(\mathrm{H}_{1}\left(\Sigma_{g}\right)\right) \hookrightarrow \mathcal{C}\left(\Sigma_{g}\right) / \mathcal{I}\left(\Sigma_{g}\right)$ induces a surjection on $\pi_{1}$.

Remark At first glance, it may appear that $\mathcal{L}^{W}\left(\mathrm{H}_{1}\left(\Sigma_{g}\right)\right)$ is contained in the cone of $\left\langle a_{g}\right\rangle$. However, while it is true that $i_{a}\left(a_{g}, x\right)=0$ for all $x \in W$, this does not imply that $\langle x\rangle$ is adjacent to $\left\langle a_{g}\right\rangle$ in $\mathcal{L}^{W}\left(\mathrm{H}_{1}\left(\Sigma_{g}\right)\right)$ (for instance, consider $x=2 a_{1}+a_{g}$ ). 
Before proving Lemma 6.3, we need a definition.

Definition Let $L$ be a 1 -dimensional summand of a $\mathbb{Z}$-module $M$ which has a fixed free basis $\left\{x_{1}, \ldots, x_{n}\right\}$. Observe that $L=\langle v\rangle$ for a primitive vector $v$ (that is, $v$ is not divisible by any integer $n \geq 2$ ) and that $v$ is unique up to multiplication by \pm 1 . Expand $v$ as

$$
v=c_{1} x_{1}+\cdots+c_{n} x_{n} .
$$

For any $1 \leq i \leq n$, we then define $\operatorname{rk}_{x_{i}}(L)=\left|c_{i}\right|$ (by the observation this is well-defined). We will call this the $x_{i}$-rank of $L$.

The proof of Lemma 6.3 then goes as follows:

Proof of Lemma 6.3 The proofs of both parts of the lemma are similar; we will prove the (slightly more difficult) second part and leave the first to the reader.

The proof will be by induction on

$$
R=\max \left\{\mathrm{rk}_{b_{g}}(A) \mid A \text { is a vertex of } \ell\right\} .
$$

The case $R=0$ being trivial, we assume that $R>0$. We first claim that we can assume that $\ell$ does not contain two adjacent vertices $A$ and $B$ so that $\operatorname{rk}_{b_{g}}(A)=\operatorname{rk}_{b_{g}}(B)=R$. Indeed, assume it does and that $A=\left\langle v_{1}\right\rangle$ and $B=\left\langle v_{2}\right\rangle$. Trivially we can assume that $A \neq B$. Replacing $v_{2}$ by $-v_{2}$ if necessary, it follows that $\operatorname{rk}_{b_{g}}\left(\left\langle v_{1}-v_{2}\right\rangle\right)=0$. Figure 6.a then shows that we can homotope (in $\mathcal{C}\left(\Sigma_{g}\right) / \mathcal{I}\left(\Sigma_{g}\right)$ ) the segment $A-B$ to $A-\left\langle v_{1}-v_{2}\right\rangle-B$, rendering $A$ and $B$ nonadjacent.

Now consider some segment $X-A-Y$ of $\ell$ so that $\operatorname{rk}_{b_{g}}(A)=R$ and $\operatorname{rk}_{b_{g}}(X), \operatorname{rk}_{b_{g}}(Y)$ $<R$. Lift $X, A, Y$, and $L$ to curves $\tilde{X}, \tilde{A}, \tilde{Y}, \tilde{L} \in \mathcal{C}_{\text {nosep }}\left(\Sigma_{g}\right)$ so that $\{\tilde{X}, \tilde{A}, \tilde{L}\}$ and $\{\tilde{Y}, \tilde{A}, \tilde{L}\}$ are simplices of $\mathcal{C}_{\text {nosep }}\left(\Sigma_{g}\right)$. Cutting $\Sigma_{g}$ along $\tilde{A} \cup \tilde{L}$, we get a copy of $\Sigma_{g-2,4}$ in which both $\tilde{X}$ and $\tilde{Y}$ are nonseparating curves. Using Lemma A.2 from the Appendix, we can find a sequence of nonseparating curves

$$
\tilde{X}=\tilde{Z}_{1}, \tilde{Z}_{2}, \ldots, \tilde{Z}_{k}=\tilde{Y}
$$

in $\Sigma_{g-2,4}$ so that for $1 \leq i<k$ we have $i_{g}\left(\tilde{Z}_{i}, \tilde{Z}_{i+1}\right)=1$. Gluing the boundary components of our $\Sigma_{g-2,4}$ back together to recover our original $\Sigma_{g}$, we have obtained a sequence of curves

$$
\tilde{X}=\tilde{Z}_{1}, \tilde{Z}_{2}, \ldots, \tilde{Z}_{k}=\tilde{Y}
$$

so that for $1 \leq i \leq k$ the set $\left\{\tilde{Z}_{i}, \tilde{A}, \tilde{L}\right\}$ is a simplex of $\mathcal{C}_{\text {nosep }}\left(\Sigma_{g}\right)$ and so that for $1 \leq i<k$ we have $i_{g}\left(\tilde{Z}_{i}, \tilde{Z}_{i+1}\right)=1$. 
Let $a$ be a primitive vector in $\mathrm{H}_{1}\left(\Sigma_{g}\right)$ so that $A=\langle a\rangle$, and for $1 \leq i \leq k$ let $z_{i}$ be a primitive vector in $\mathrm{H}_{1}\left(\Sigma_{g}\right)$ so that $Z_{i}:=\left\langle z_{i}\right\rangle$ is the projection of $\tilde{Z}_{i}$ to $\mathcal{L}\left(\mathrm{H}_{1}\left(\Sigma_{g}\right)\right)$. By the division algorithm, we can find integers $q_{i}$ (with $q_{1}=q_{k}=0$ ) so that

$$
\operatorname{rk}_{b_{g}}\left(\left\langle z_{i}+q_{i} a\right\rangle\right)<R .
$$

Set $z_{i}^{\prime}=z_{i}+q_{i} a$ and $Z_{i}^{\prime}=\left\langle z_{i}^{\prime}\right\rangle$ (hence $Z_{1}^{\prime}=Z_{1}=X$ and $Z_{k}^{\prime}=Z_{k}=Y$ ). Observe that for all $1 \leq i \leq k$, the set $\left\{Z_{i}^{\prime}, A, L\right\}$ is still a simplex of $\mathcal{L}\left(\mathrm{H}_{1}\left(\Sigma_{g}\right)\right)$, and in addition we still have for all $1 \leq i<k$ that $i_{a}\left(z_{i}^{\prime}, z_{i+1}^{\prime}\right)=1$. We can therefore lift the $Z_{i}^{\prime}$ to nonseparating curves $\tilde{Z}_{i}^{\prime}$ in $\Sigma_{g}$ which are disjoint from $\tilde{A}$ and $\tilde{L}$ and which satisfy $i_{g}\left(\tilde{Z}_{i}^{\prime}, \tilde{Z}_{i+1}^{\prime}\right)=1$ for all $1 \leq i<k$.

For $1 \leq i<k$, let $\tilde{S}_{i}$ be the boundary component of a regular neighborhood of $\tilde{Z}_{i} \cup \tilde{Z}_{i+1}$. Hence $\tilde{S}_{i}$ is a separating curve disjoint from $\tilde{Z}_{i}, \tilde{Z}_{i+1}, \tilde{A}$, and $\tilde{L}$. For $1 \leq i<k$, cutting $\Sigma_{g}$ along $\tilde{S}_{i}$ and $L$ yields a copy of $\Sigma_{g-2,3}$. A simple dimension count shows that there must be some simple closed nonseparating curve $\tilde{B}_{i}$ in this copy of $\Sigma_{g-2,3}$ whose homology class lies in $W$ (and, in particular, the span of whose homology class has $b_{g}$-rank 0 ). Observe that the path

$$
\tilde{Z}_{1}-\tilde{A}-\tilde{Z}_{k}
$$

in $\mathcal{C}\left(\Sigma_{g}\right)$ is homotopic (fixing the endpoints) to

$$
\tilde{Z}_{1}-\tilde{S}_{1}-\tilde{Z}_{2}-\tilde{S}_{2}-\cdots-\tilde{S}_{k-1}-\tilde{Z}_{k},
$$

which is then homotopic to

$$
\tilde{Z}_{1}-\tilde{B}_{1}-\tilde{Z}_{2}-\tilde{B}_{2}-\cdots-\tilde{B}_{k-1}-\tilde{Z}_{k}
$$

Projecting this homotopy down to $\operatorname{link}_{L}\left(\mathcal{C}\left(\Sigma_{g}\right) / \mathcal{I}\left(\Sigma_{g}\right)\right)$ allows us to homotope the segment $X-A-Y$ to a new segment which lies in $\operatorname{link}_{L}\left(\mathcal{L}\left(\mathrm{H}_{1}\left(\Sigma_{g}\right)\right)\right)$ and which does not contain any vertices whose $b_{g}$-rank is greater than or equal to $R$. Repeating this process allows us to remove all vertices of $\ell$ whose $b_{g}$-rank equals $R$, and we are done by induction.

\subsection{Completing the proof for $g \geq 3$}

In this section, we complete the proof of Theorem 5.3 for $g \geq 3$. Define

$$
W^{\prime}=\left\langle a_{1}, b_{1}, \ldots, a_{g-1}, b_{g-1}\right\rangle .
$$

We will prove the following:

Lemma 6.5 The inclusion $\mathcal{L}^{W^{\prime}}\left(\mathrm{H}_{1}\left(\Sigma_{g}\right)\right) \hookrightarrow \mathcal{C}\left(\Sigma_{g}\right) / \mathcal{I}\left(\Sigma_{g}\right)$ induces a surjection on $\pi_{1}$. 
First, though, we will use Lemma 6.5 to prove the desired special case of Theorem 5.3.

Proof of Theorem 5.3 for $\boldsymbol{g} \geq \mathbf{3}$ By Lemma 6.5, it is enough to show that the inclusion $\mathcal{L}^{W^{\prime}}\left(\mathrm{H}_{1}\left(\Sigma_{g}\right)\right) \hookrightarrow \mathcal{C}\left(\Sigma_{g}\right) / \mathcal{I}\left(\Sigma_{g}\right)$ induces the zero map on $\pi_{1}$. Indeed, the inclusion map $\mathcal{L}^{W^{\prime}}\left(\mathrm{H}_{1}\left(\Sigma_{g}\right)\right) \hookrightarrow \mathcal{L}^{W}\left(\mathrm{H}_{1}\left(\Sigma_{g}\right)\right)$ induces the zero map on $\pi_{1}$, as $\mathcal{L}^{W^{\prime}}\left(\mathrm{H}_{1}\left(\Sigma_{g}\right)\right)$ can be contracted in $\mathcal{L}^{W}\left(\mathrm{H}_{1}\left(\Sigma_{g}\right)\right)$ to $\left\langle a_{g}\right\rangle$.

We now prove Lemma 6.5.

Proof of Lemma 6.5 Fix a basepoint in $\mathcal{C}\left(\Sigma_{g}\right) / \mathcal{I}\left(\Sigma_{g}\right)$ which lies in the image of $\mathcal{L}^{W^{\prime}}\left(\mathrm{H}_{1}\left(\Sigma_{g}\right)\right)$, and consider a based loop $\ell$, which we can assume to be a simplicial loop in the 1-skeleton. By Lemma 6.3, we can assume that $\ell$ lies in the image of $\mathcal{L}^{W}\left(\mathrm{H}_{1}\left(\Sigma_{g}\right)\right)$. We will prove that $\ell$ can be homotoped into the image of $\mathcal{L}^{W^{\prime}}\left(\mathrm{H}_{1}\left(\Sigma_{g}\right)\right)$ by induction on

$$
R=\max \left\{\operatorname{rk}_{a_{g}}(A) \mid A \text { is a vertex of } \ell\right\} .
$$

The case $R=0$ being trivial, we assume that $R>0$. By an argument like that in the proof of Lemma 6.3, we can assume that no two adjacent vertices in $\ell$ have $a_{g}$-rank equal to $R$. Now consider a subpath $X-A-Y$ of $\ell$ so that $\operatorname{rk}_{a_{g}}(A)=R$ and $\operatorname{rk}_{a_{g}}(X), \operatorname{rk}_{a_{g}}(Y)<R$. Since $\operatorname{link}_{A}\left(\mathcal{L}\left(\mathrm{H}_{1}\left(\Sigma_{g}\right)\right)\right.$ is connected (this is our main use of the assumption $g \geq 3$ ), we can find a path

$$
X=Z_{1}-Z_{2}-\cdots-Z_{k}=Y
$$

in $\operatorname{link}_{A}\left(\mathcal{L}\left(\mathrm{H}_{1}\left(\Sigma_{g}\right)\right)\right)$, which by Lemma 6.3 we can assume lies in $\mathcal{L}^{W}\left(\mathrm{H}_{1}\left(\Sigma_{g}\right)\right)$. Let $a$ be a primitive vector in $\mathrm{H}_{1}\left(\Sigma_{g}\right)$ so that $A=\langle a\rangle$, and for $1 \leq i \leq k$ let $z_{i}$ be a primitive vector in $\mathrm{H}_{1}\left(\Sigma_{g}\right)$ so that $Z_{i}=\left\langle z_{i}\right\rangle$. By the division algorithm, for $1 \leq i \leq k$ there exists an integer $q_{i}$ (with $q_{1}=q_{k}=0$ ) so that $\mathrm{rk}_{a_{g}}\left(\left\langle z_{i}+q_{i} a\right\rangle\right)<R$. Setting $Z_{i}^{\prime}=\left\langle z_{i}+q_{i} a\right\rangle$, the path

$$
X=Z_{1}^{\prime}-Z_{2}^{\prime}-\cdots-Z_{k}^{\prime}=Y
$$

still lies in $\mathcal{L}^{W}\left(\mathrm{H}_{1}\left(\Sigma_{g}\right)\right) \cap \operatorname{link}_{A}\left(\mathcal{L}\left(\mathrm{H}_{1}\left(\Sigma_{g}\right)\right)\right)$. We can therefore homotope $X-A-Y$ to this path, eliminating $A$ without introducing any new vertices whose $a_{g}$-rank is greater than or equal to $R$. Repeating this process, we can eliminate all vertices whose $a_{g}$-rank equals $R$, and we are done by induction.

\subsection{Completing the proof for $g=2$}

In genus 2 , the above proof fails. To complete the proof in this case, we introduce one final object. 


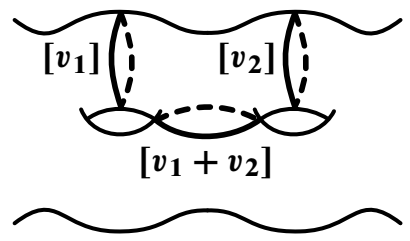

a

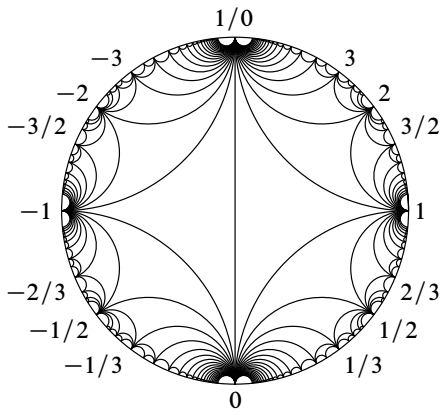

b

Figure 6: a: Illustration that triangles $\left\langle v_{1}\right\rangle-\left\langle \pm v_{1} \pm v_{2}\right\rangle-\left\langle v_{2}\right\rangle$ in $\mathcal{L}\left(\mathrm{H}_{1}\left(\Sigma_{g}\right)\right)$ come from triangles in $\mathcal{C}\left(\Sigma_{g}\right)$

b: The Farey tessellation

Definition Let $V$ be a $\mathbb{Z}$-module with a symplectic inner product $i(\cdot, \cdot)$. The augmented complex of unimodular isotropic lines in $V$, which we will denote $\overline{\mathcal{L}}(V)$, is equal to $\mathcal{L}(V)$ with 2-cells attached to all triangles of the form

$$
\left\langle v_{1}\right\rangle-\left\langle \pm v_{1} \pm v_{2}\right\rangle-\left\langle v_{2}\right\rangle
$$

for all edges $\left\{\left\langle v_{1}\right\rangle,\left\langle v_{2}\right\rangle\right\}$ of $\mathcal{L}(V)$. For any submodule $W$ of $V$, we also define $\overline{\mathcal{L}}^{W}(V)$ to be the full subcomplex of $\overline{\mathcal{L}}(V)$ spanned by vertices $L$ with $L \subset W$.

Figure 6.a yields the following lemma:

Lemma 6.6 The inclusion

$$
\mathcal{L}\left(\mathrm{H}_{1}\left(\Sigma_{g}\right)\right) \hookrightarrow \mathcal{C}\left(\Sigma_{g}\right) / \mathcal{I}\left(\Sigma_{g}\right)
$$

extends to a map

$$
\overline{\mathcal{L}}\left(\mathrm{H}_{1}\left(\Sigma_{g}\right)\right) \hookrightarrow \mathcal{C}\left(\Sigma_{g}\right) / \mathcal{I}\left(\Sigma_{g}\right)
$$

We now prove the following (recall that the Farey tessellation of $\mathbb{H}^{2}$ is the 2-dimensional flag complex whose vertices are elements of $\mathbb{Q} \cup\{\infty\}$ and where $b / a$ and $d / c$ are adjacent if $|a d-b c|=1$; cf Figure 6.b):

Lemma 6.7 Let $W$ be a maximal isotropic subspace of $\mathrm{H}_{1}\left(\Sigma_{2}\right)$. Then the simplicial complex $\overline{\mathcal{L}}^{W}\left(\mathrm{H}_{1}\left(\Sigma_{2}\right)\right)$ is homeomorphic to the Farey tessellation of $\mathbb{H}^{2}$ (with the weak topology). In particular, $\overline{\mathcal{L}}^{W}\left(\mathrm{H}_{1}\left(\Sigma_{2}\right)\right)$ is contractible.

Proof Identifying $W$ with $\mathbb{Z}^{2}$, observe that $\overline{\mathcal{L}}^{W}\left(\mathrm{H}_{1}\left(\Sigma_{2}\right)\right)$ equals the following simplicial complex: 
- The vertices are the 1 -dimensional summands of $\mathbb{Z}^{2}$. These are classified by their slope in $\mathbb{Q} \cup\{\infty\}$.

- Two vertices $b / a, d / c \in \mathbb{Q} \cup \infty$ form a 1 -simplex if $\langle(a, b),(c, d)\rangle=\mathbb{Z}^{2}$. This is true if and only if the matrix whose columns are the vectors $(a, b)$ and $(c, d)$ is invertible over $\mathbb{Z}$; ie, if and only if the determinant $a d-b c$ equals \pm 1 .

- Three vertices $b / a, d / c$, and $f / e$ form a 2-simplex if $b / a$ and $d / c$ form an edge and $(e, f)= \pm(a, b) \pm(c, d)$. It is easy to see that this is true if and only if each pair of vertices form an edge.

The claim follows.

We now prove Theorem 5.3 when $g=2$.

Proof of Theorem 5.3 for $\boldsymbol{g}=2$ Consider a loop $\ell$ in $\mathcal{C}\left(\Sigma_{2}\right) / \mathcal{I}\left(\Sigma_{2}\right)$ based at

$$
\left\langle a_{1}\right\rangle \in \overline{\mathcal{L}}\left(\mathrm{H}_{1}\left(\Sigma_{2}\right)\right) \subset \mathcal{C}\left(\Sigma_{2}\right) / \mathcal{I}\left(\Sigma_{2}\right) .
$$

We can assume that $\ell$ is a simplicial loop in the $1-$ skeleton. By Lemma 6.3, we can also assume that $\ell$ lies in $\overline{\mathcal{L}}^{\left\langle a_{1}, b_{1}, a_{2}\right\rangle}\left(\mathrm{H}_{1}\left(\Sigma_{2}\right)\right)$. We will prove that $\ell$ can be homotoped to a point by induction on the length of $\ell$. Observe first that if $\ell$ lies in $\overline{\mathcal{L}}^{\left\langle a_{1}, a_{2}\right\rangle}\left(\mathrm{H}_{1}\left(\Sigma_{2}\right)\right)$, then Lemma 6.7 implies that $\ell$ can be contracted to a point. Assume, therefore, that $\ell$ contains a vertex whose $b_{1}$-rank is nonzero. Let $\ell^{\prime}$ be a subpath of $\ell$ all of whose vertices have $b_{1}$-rank nonzero and which is maximal with respect to this property. In addition, let $x$ and $y$ be the vertices of $\ell$ immediately preceding and immediately succeeding $\ell^{\prime}$; in other words, $\ell$ contains the subpath

$$
x-\ell^{\prime}-y .
$$

Observe that by the maximality of $\ell^{\prime}$, both $x$ and $y$ have $b_{1}$-rank equal to 0 ; ie, $x, y \in \overline{\mathcal{L}}^{\left\langle a_{1}, a_{2}\right\rangle}$. Additionally, since every vertex of $\ell^{\prime}$ has positive $b_{1}$-rank, both $x$ and $y$ must have $a_{1}$-rank 0 . We conclude that $x=y=\left\langle a_{2}\right\rangle$. In other words,

$$
x-\ell^{\prime}-y
$$

is a loop. If $\ell^{\prime}$ consists of a single vertex, then we can contract this loop and thus shorten $\ell$, and we are done. Otherwise, since every vertex of $\ell^{\prime}$ has $b_{1}$-rank greater than 0 , it follows that every vertex of $\ell^{\prime}$ has $a_{1}$-rank equal to 0 . We conclude that the loop

lies in $\overline{\mathcal{L}}^{\left\langle a_{2}, b_{1}\right\rangle}\left(\mathrm{H}_{1}\left(\Sigma_{2}\right)\right)$. By Lemma 6.7, we can contract this loop to a point, thus shortening $\ell$, and we are done. 


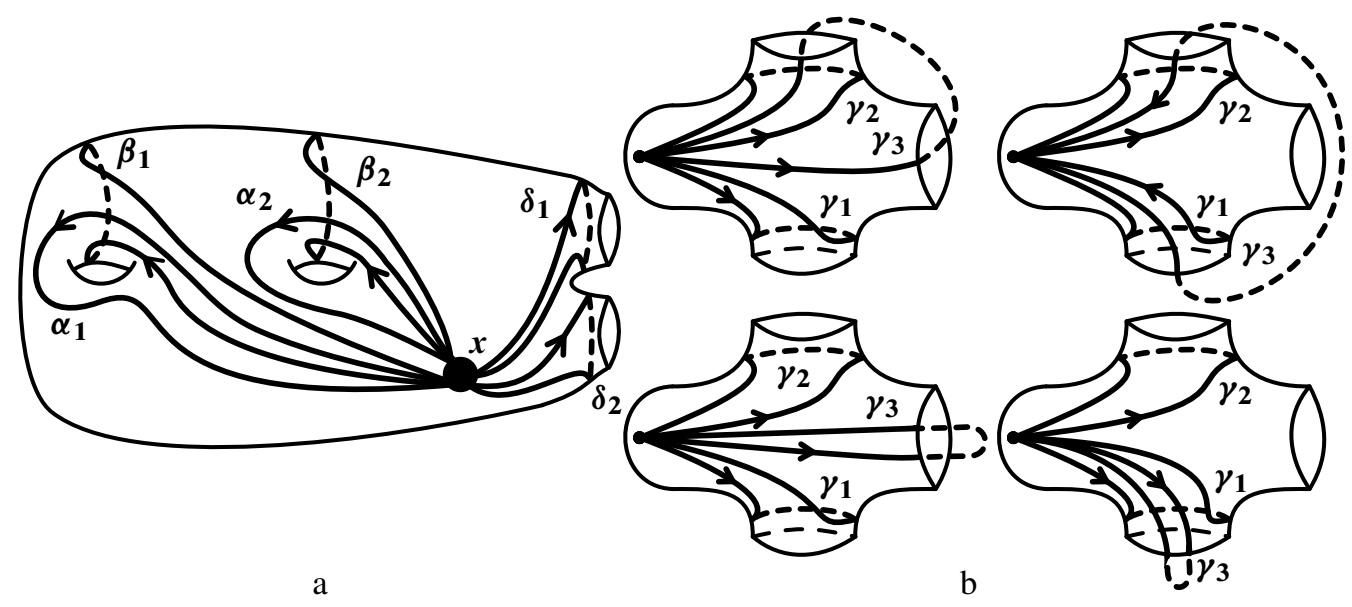

Figure 7: a: Generators for $\pi_{1}\left(\Sigma_{2,2}, x\right)$

b: The four configurations of curves on three-holed spheres needed

\section{Appendix A Appendix on surface topology}

In this appendix, we will prove three lemmas about the topology of surfaces for which we are unable to provide appropriate references, though they are certainly known to the experts.

\section{A.1 Generators for the commutator subgroup of a surface group}

In this section, we will prove a lemma which provides generators for the commutator subgroup of a surface group. It is a generalization of a lemma which appears in a paper of Johnson [15, Lemma 7]. It also is implicit in the unpublished thesis of van den Berg [20, Proposition 2.4.2], though her proof is not quite complete.

Lemma A.1 Let $g \geq 1$ and $\pi^{\prime}=\left[\pi_{1}\left(\Sigma_{g, n}, x\right), \pi_{1}\left(\Sigma_{g, n}, x\right)\right]$. Assume that the basepoint $x$ is in the interior of $\Sigma_{g, n}$. Then $\pi^{\prime}$ is generated by $\left[\gamma_{1}, \gamma_{2}\right]$, where $\gamma_{1}, \gamma_{2} \in \pi_{1}\left(\Sigma_{g, n}, x\right)$ are simple closed curves so that $\gamma_{1} \cap \gamma_{2}=\{x\}$ and so that a regular neighborhood of $\gamma_{1} \cup \gamma_{2}$ is homeomorphic to a one-holed torus.

Proof Let $\Gamma$ be the subgroup generated by the indicated elements. We will first prove that $\Gamma$ contains $\left[\gamma_{1}, \gamma_{2}\right]$ for simple closed curves $\gamma_{1}$ and $\gamma_{2}$ so that $\gamma_{1} \cap \gamma_{2}=\{x\}$ and so that a regular neighborhood of $\gamma_{1} \cup \gamma_{2}$ is homeomorphic to a three-holed sphere. There are two cases. In the first, one of the $\gamma_{i}$ (say $\gamma_{2}$ ) is nonseparating. We can then find a simple closed curve $\gamma_{3}$ so that $\gamma_{3} \cap \gamma_{1}=\gamma_{3} \cap \gamma_{2}=\{x\}$ and so that regular 
neighborhoods of both $\gamma_{1} \gamma_{3} \cup \gamma_{2}$ and $\gamma_{3} \cup \gamma_{2}$ are homeomorphic to one-holed tori (see the top portion of Figure 7.b). We then have the identity

$$
\left[\gamma_{1}, \gamma_{2}\right]=\left(\gamma_{3}\left[\gamma_{1} \gamma_{3}, \gamma_{2}\right] \gamma_{3}^{-1}\right)\left(\gamma_{3}\left[\gamma_{2}, \gamma_{3}\right] \gamma_{3}^{-1}\right)
$$

Observe that conjugation does not change the "type" of a commutator (this follows from the identification of $\pi_{1}\left(\Sigma_{g, n}, x\right)$ with the kernel in the Birman exact sequence, and is the reason we require the basepoint to be in the interior). Hence the right hand side is in $\Gamma$, so the left hand side is as well.

In the second case, both of the $\gamma_{i}$ are separating curves. Reordering the $\gamma_{i}$ if necessary, we can then find a simple closed nonseparating curve $\gamma_{3}$ so that $\gamma_{3} \cap \gamma_{1}=\gamma_{3} \cap \gamma_{2}=\{x\}$ and so that $\gamma_{1} \gamma_{3}$ is nonseparating (see the bottom portion of Figure 7.b; this is where we use the assumption that $g \geq 1$ ). We again have the identity

$$
\left[\gamma_{1}, \gamma_{2}\right]=\left(\gamma_{3}\left[\gamma_{1} \gamma_{3}, \gamma_{2}\right] \gamma_{3}^{-1}\right)\left(\gamma_{3}\left[\gamma_{2}, \gamma_{3}\right] \gamma_{3}^{-1}\right)
$$

By the previous case, the right hand side is in $\Gamma$, so the left hand side is as well.

Now let

$$
S=\left\{\alpha_{1}, \beta_{1}, \ldots, \alpha_{g}, \beta_{g}, \delta_{1}, \ldots, \delta_{n}\right\}
$$

be a standard basis for $\pi_{1}\left(\Sigma_{g, n}, x\right)$ (see Figure 7.a). Thus $\pi^{\prime}$ is normally generated by $\left[\gamma_{1}, \gamma_{2}\right]$ for $\gamma_{1}, \gamma_{2} \in S$. Since we have proven that every such commutator is in $\Gamma$, we conclude that $\Gamma=\pi^{\prime}$, as desired.

\section{A.2 A connectedness lemma}

In this section, we prove a lemma which allows us to move between simple closed nonseparating curves in a simple manner.

Lemma A.2 For $g \geq 1$ and $n \geq 0$, let $\gamma$ and $\gamma^{\prime}$ be two simple closed nonseparating curves in $\Sigma_{g, n}$. We can then find a sequence $\gamma_{1}, \gamma_{2}, \ldots, \gamma_{k}$ of simple closed nonseparating curves in $\Sigma_{g, n}$ so that $\gamma_{1}=\gamma, \gamma_{k}=\gamma^{\prime}$, and so that for $1 \leq i<k$ we have $i_{g}\left(\gamma_{i}, \gamma_{i+1}\right)=1$.

Remark For $n=0$, this lemma is well-known. However, we need it for surfaces with boundary, so we include a proof.

Proof of Lemma A.2 It is well-known that there exists a set $S$ of simple closed curves in $\Sigma_{g, n}$ so that $\left\{T_{\delta} \mid \delta \in S\right\}$ generates $\operatorname{Mod}\left(\Sigma_{g, n}\right)$ and so that for all $\delta \in S$ we have $i_{g}(\delta, \gamma) \leq 1$ (for example, $S$ could be the curves the twists about which form the generating set in [5] and $\gamma$ could be the "central" curve $b$ from that paper). Observe 


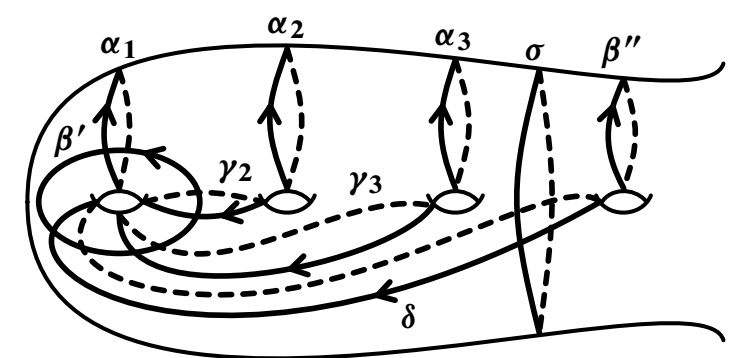

Figure 8: The various curves needed in the proof of Lemma A.3

that for all $\delta \in S$, either $T_{\delta}^{ \pm 1}(\gamma)=\gamma$ or $i_{g}\left(T_{\delta}^{ \pm 1}(\gamma), \gamma\right)=1 . \operatorname{Now}, \operatorname{Mod}\left(\Sigma_{g, n}\right)$ acts transitively on the set of simple closed nonseparating curves, so there exists a sequence of curves $\delta_{1}, \ldots, \delta_{k} \in S$ and a sequence of numbers $e_{1}, \ldots, e_{k} \in\{ \pm 1\}$ so that $T_{\delta_{1}}^{e_{1}} \cdots T_{\delta_{k}}^{e_{k}}(\gamma)=\gamma^{\prime}$. We conclude that after eliminating repetitions, the following is the desired sequence:

$$
\gamma, T_{\delta_{1}}^{e_{1}}(\gamma), T_{\delta_{1}}^{e_{1}} T_{\delta_{2}}^{e_{2}}(\gamma), \ldots, T_{\delta_{1}}^{e_{1}} T_{\delta_{2}}^{e_{2}} \cdots T_{\delta_{k}}^{e_{k}}(\gamma)
$$

\section{A.3 Realizing homology bases}

In this section, we prove a lemma which allows us to realize symplectic bases for $\mathrm{H}_{1}\left(\Sigma_{g}\right)$ in a nice manner.

Lemma A.3 Let $\left\{a_{1}, \ldots, a_{g}, b_{1}, \ldots, b_{g}\right\}$ be a symplectic basis for $\mathrm{H}_{1}\left(\Sigma_{g}\right)$, and for integers $0 \leq h, k \leq g$ let $\left\{\alpha_{1}, \ldots, \alpha_{h}, \beta_{1}, \ldots, \beta_{k}\right\}$ be a set of oriented simple closed curves in $\Sigma_{g}$ so that the following hold for all $i$ and $j$ for which the expressions are defined:

$$
\begin{array}{r}
{\left[\alpha_{i}\right]=a_{i} \text { and }\left[\beta_{i}\right]=b_{i},} \\
i_{g}\left(\alpha_{i}, \alpha_{j}\right)=0 \text { and } i_{g}\left(\beta_{i}, \beta_{j}\right)=0, \\
i_{g}\left(\alpha_{i}, \beta_{j}\right)=\delta_{i j} .
\end{array}
$$

Then we can find simple closed curves $\left\{\alpha_{h+1}, \ldots, \alpha_{g}, \beta_{k+1}, \ldots, \beta_{g}\right\}$ so that these expressions continue to hold.

Proof The proof will be by induction on $g$. The case $g=0$ is trivial. Now assume that $g>0$. If both $\alpha_{1}$ and $\beta_{1}$ are given to us, let $N$ be a small regular neighborhood of $\alpha_{1} \cup \beta_{1}$. Observe that $N$ is a copy of $\Sigma_{1,1}$ which is disjoint from the remaining 
$\alpha_{i}$ and $\beta_{j}$. Hence $\Sigma_{g} \backslash N$ is a copy of $\Sigma_{g-1,1}$ containing $\left\{\alpha_{2}, \ldots, \alpha_{h}, \beta_{2}, \ldots, \beta_{k}\right\}$. Let $\Sigma_{g} \backslash N \longrightarrow \Sigma_{g-1}$ be the embedding induced by gluing a disk to the boundary component. By induction, we can solve the resulting problem on $\Sigma_{g-1}$, and it is clear that any lift of the solution to $\Sigma_{g}$ solves the problem there as well.

We can therefore assume without loss of generality that we are given no $\beta_{i}$ 's. If no curves are given, then it is trivial to find $\alpha_{1}$, so we can also assume without loss of generality that we are given $\left\{\alpha_{1}, \ldots, \alpha_{h}\right\}$ for some $h \geq 1$. Let $\sigma$ be any separating curve dividing $\Sigma_{g}$ into two subsurfaces $S_{1}$ and $S_{2}$ with $\alpha_{i} \in S_{1}$ for all $i$. Arrange the $\alpha_{i}$ and $\sigma$ in the pattern indicated in Figure 8, and let $\gamma_{2}, \ldots, \gamma_{h}$ and $\beta^{\prime}$ be the curves indicated there. It is clear that with the indicated orientations we have

$$
\left[\gamma_{i}\right]=\left[\alpha_{i}\right]-\left[\alpha_{1}\right]
$$

Also, $\sigma$ induces a symplectic splitting

$$
\mathrm{H}_{1}\left(\Sigma_{g}\right)=\mathrm{H}_{1}\left(S_{1}\right) \oplus \mathrm{H}_{1}\left(S_{2}\right)
$$

Pick $d \in \mathbb{Z}$ and an irreducible vector $b^{\prime \prime} \in \mathrm{H}_{1}\left(S_{2}\right)$ so that the projection of $b_{1}$ to $\mathrm{H}_{1}\left(S_{2}\right)$ equals $d b^{\prime \prime}$ (if this projection is 0 , then $d=0$ and $b^{\prime \prime}$ is arbitrary). Let $\beta^{\prime \prime}$ be any simple closed curve in $S_{2}$ realizing $b^{\prime \prime}$. Since $i_{a}\left(a_{1}, b_{1}\right)=1$, we can find $c_{1}, \ldots, c_{h} \in \mathbb{Z}$ so that

$$
b_{1}=\left[\beta^{\prime}\right]+\left(\sum_{i=1}^{h} c_{i} a_{i}\right)+d\left[\beta^{\prime \prime}\right] .
$$

See Figure 8 . Let $\delta$ be the curve indicated there. Hence

$$
[\delta]=\left[\beta^{\prime \prime}\right]-\left[\alpha_{1}\right] .
$$

Set

$$
\beta_{1}=T_{\alpha_{1}}^{c_{1}+\cdots+c_{h}+d} T_{\gamma_{2}}^{-c_{2}} \cdots T_{\gamma_{h}}^{-c_{h}} T_{\delta}^{-d}\left(\beta^{\prime}\right) .
$$

Observe that $i_{g}\left(\alpha_{i}, \beta_{1}\right)=\delta_{i 1}$. Also,

$$
\begin{aligned}
{\left[\beta_{1}\right]=} & {\left[\beta^{\prime}\right]+\left(c_{1}+\cdots+c_{h}+d\right)\left[\alpha_{1}\right]+c_{2}\left[\gamma_{2}\right]+\cdots+c_{h}\left[\gamma_{h}\right]+d[\delta] } \\
= & {\left[\beta^{\prime}\right]+\left(c_{1}+\cdots+c_{h}+d\right)\left[\alpha_{1}\right] } \\
& +c_{2}\left(\left[\alpha_{i}\right]-\left[\alpha_{1}\right]\right)+\cdots+c_{h}\left(\left[\alpha_{h}\right]-\left[\alpha_{1}\right]\right)+d\left(\left[\beta^{\prime \prime}\right]-\left[\alpha_{1}\right]\right) \\
= & {\left[\beta^{\prime}\right]+\left(c_{1} a_{1}+\cdots+c_{h} a_{h}\right)+d\left[\beta^{\prime \prime}\right]=b_{1}, }
\end{aligned}
$$

as desired. This reduces us to a previous case, and completes the proof. 
Andrew Putman

\section{References}

[1] M A Armstrong, On the fundamental group of an orbit space, Proc. Cambridge Philos. Soc. 61 (1965) 639-646 MR0187244

[2] J S Birman, Mapping class groups and their relationship to braid groups, Comm. Pure Appl. Math. 22 (1969) 213-238 MR0243519

[3] J S Birman, On Siegel's modular group, Math. Ann. 191 (1971) 59-68 MR0280606

[4] J S Birman, Braids, links, and mapping class groups, Annals of Mathematics Studies 82, Princeton University Press (1974) MR0375281

[5] S Gervais, A finite presentation of the mapping class group of a punctured surface, Topology 40 (2001) 703-725 MR1851559

[6] P Gold, On the mapping class and symplectic modular group, $\mathrm{PhD}$ thesis, New York University (1961)

[7] A J Hahn, O T O'Meara, The classical groups and $K$-theory, with a foreword by J Dieudonné, Grundlehren series 291, Springer (1989) MR1007302

[8] R M Hain, Torelli groups and geometry of moduli spaces of curves, from: "Current topics in complex algebraic geometry (Berkeley, CA, 1992/93)”, Math. Sci. Res. Inst. Publ. 28, Cambridge Univ. Press (1995) 97-143 MR1397061

[9] J L Harer, Stability of the homology of the mapping class groups of orientable surfaces, Ann. of Math. (2) 121 (1985) 215-249 MR786348

[10] W J Harvey, Geometric structure of surface mapping class groups, from: "Homological group theory (Proc. Sympos., Durham, 1977)", (C T C Wall, editor), London Math. Soc. Lecture Note Ser. 36, Cambridge Univ. Press (1979) 255-269 MR564431

[11] N V Ivanov, Complexes of curves and Teichmüller modular groups, Uspekhi Mat. Nauk 42 (1987) 49-91, 255 MR896878

[12] N V Ivanov, Subgroups of Teichmüller modular groups, Translations of Mathematical Monographs 115, Amer. Math. Soc. (1992) MR1195787 Translated from the Russian by EJF Primrose and revised by the author

[13] D L Johnson, A survey of the Torelli group, from: "Low-dimensional topology (San Francisco, CA, 1981)”, Contemp. Math. 20, Amer. Math. Soc. (1983) 165-179 MR718141

[14] D L Johnson, The structure of the Torelli group. I. A finite set of generators for $\mathcal{I}$, Ann. of Math. (2) 118 (1983) 423-442 MR727699

[15] D L Johnson, The structure of the Torelli group. II. A characterization of the group generated by twists on bounding curves, Topology 24 (1985) 113-126 MR793178

[16] D L Johnson, The structure of the Torelli group. III. The abelianization of $\mathcal{T}$, Topology 24 (1985) 127-144 MR793179 
[17] H Klingen, Charakterisierung der Siegelschen Modulgruppe durch ein endliches System definierender Relationen, Math. Ann. 144 (1961) 64-82 MR0133303

[18] W Magnus, Über n-dimensionale Gittertransormationen, Acta. Math. 64 (1934) 353367

[19] J Powell, Two theorems on the mapping class group of a surface, Proc. Amer. Math. Soc. 68 (1978) 347-350 MR0494115

[20] B van den Berg, On the Abelianization of the Torelli group, $\mathrm{PhD}$ thesis, University of Utrecht (2003)

Department of Mathematics, University of Chicago

5734 University Ave, Chicago, Il 60637, USA

andyp@math.uchicago.edu

http: //www.math.uchicago.edu/ andyp

Proposed: Joan Birman

Seconded: Walter Neumann, Martin Bridson
Received: 25 August 2006

Accepted: 10 April 2007 